\title{
Three-micron spectra of AGB stars and supergiants in nearby galaxies ${ }^{\star}$
}

\author{
M. Matsuura ${ }^{1}$, A. A. Zijlstra ${ }^{1}$, J. Th. van Loon $^{2}$, I. Yamamura ${ }^{3}$, A. J. Markwick ${ }^{4}$, P. A. Whitelock ${ }^{5}$, P. M. Woods ${ }^{1}$, \\ J. R. Marshall ${ }^{2}$, M. W. Feast ${ }^{6}$, and L. B. F. M. Waters ${ }^{7,8}$ \\ 1 School of Physics and Astronomy, University of Manchester, Sackville Street, PO Box 88, Manchester M60 1QD, UK \\ e-mail: m.matsuura@manchester.ac.uk \\ 2 Astrophysics Group, School of Chemistry and Physics, Keele University, Staffordshire ST5 5BG, UK \\ 3 The Institute of Space and Astronautical Science, Japan Aerospace Exploration Agency, Yoshino-dai 3-1-1, Sagamihara, \\ Kanagawa 229-8510, Japan \\ ${ }^{4}$ Space Science Division, NASA Ames Research Centre, MS 245-3, Moffett Field, CA 94035, USA \\ 5 South African Astronomical Observatory, PO Box 9, 7935 Observatory, South Africa \\ 6 Astronomy Department, University of Cape Town, 7701 Rondebosch, South Africa \\ 7 Astronomical Institute "Anton Pannekoek”, University of Amsterdam, Kruislaan 403, 1098 SJ, Amsterdam, The Netherlands \\ ${ }^{8}$ Instituut voor Sterrenkunde, Katholieke Universiteit Leuven, Celestijnenlaan 200B, 3001 Heverlee, Belgium
}

Received 3 November 2004 / Accepted 21 December 2004

\begin{abstract}
The dependence of stellar molecular bands on the metallicity is studied using infrared $L$-band spectra of AGB stars (both carbon-rich and oxygen-rich) and M-type supergiants in the Large and Small Magellanic Clouds (LMC and SMC) and in the Sagittarius Dwarf Spheroidal Galaxy. The spectra cover SiO bands for oxygen-rich stars, and acetylene $\left(\mathrm{C}_{2} \mathrm{H}_{2}\right), \mathrm{CH}$ and $\mathrm{HCN}$ bands for carbon-rich AGB stars. The equivalent width of acetylene is found to be high even at low metallicity. The high $\mathrm{C}_{2} \mathrm{H}_{2}$ abundance can be explained with a high carbon-to-oxygen $(\mathrm{C} / \mathrm{O})$ ratio for lower metallicity carbon stars. In contrast, the HCN equivalent width is low: fewer than half of the extra-galactic carbon stars show the $3.5 \mu \mathrm{m} \mathrm{HCN}$ band, and only a few LMC stars show high HCN equivalent width. HCN abundances are limited by both nitrogen and carbon elemental abundances. The amount of synthesized nitrogen depends on the initial mass, and stars with high luminosity (i.e. high initial mass) could have a high HCN abundance. $\mathrm{CH}$ bands are found in both the extra-galactic and Galactic carbon stars. One SMC post-AGB star, SMC-S2, shows the $3.3 \mu \mathrm{m}$ PAH band. This first detection of a PAH band from an SMC post-AGB star confirms PAHs can form in these low-metallicity stars. None of the oxygen-rich LMC stars show SiO bands, except one possible detection in a low quality spectrum. The limits on the equivalent widths of the SiO bands are below the expectation of up to $30 \AA$ for LMC metallicity. Several possible explanations are discussed, mostly based on the effect of pulsation and circumstellar dust. The observations imply that LMC and SMC carbon stars could reach mass-loss rates as high as their Galactic counterparts, because there are more carbon atoms available and more carbonaceous dust can be formed. On the other hand, the lack of $\mathrm{SiO}$ suggests less dust and lower mass-loss rates in low-metallicity oxygen-rich stars. The effect on the ISM dust enrichment is discussed.
\end{abstract}

Key words. stars: AGB and post-AGB - infrared: stars - galaxies: stellar content - stars: atmospheres

\section{Introduction}

Asymptotic Giant Branch (AGB) and Red Supergiant (RSG) stars are important sources of dust and gas in galaxies. Within the final $10^{4}-10^{5}$ years of the AGB phase, stars lose up to $80 \%$ of their mass via a slow wind with mass-loss rates up to $10^{-4} M_{\odot} \mathrm{yr}^{-1}$. Red supergiants can experience a similar massloss phase. AGB stars have initial masses of $1-8 M_{\odot}$, but their

\footnotetext{
* Based on observations at the European Southern Observatory, on the Very Large Telescope with an instrument, ISAAC (projects 68.D-0660, 69.D-0623).
}

large population compensates for the lower mass. The other main source of dust and gas, supernovae, produce higher elements per event but occur at a much lower rate. The balance between dust production by supernovae and by AGB/RSG stars is not well known. If the mass-loss rate of AGB/RSG stars is much lower at low-metallicity (Zijlstra 2004), the relative contributions may be strongly dependent on metallicity (Edmunds 2001).

How mass-loss rates are affected by metallicity is not well understood. In general, the elemental abundances of extragalactic stars are assumed to scale with the metallicity, but this 
ignores the elements which are synthesized in the AGB stars or supergiants themselves. The wind is dust-driven, and dust forms out of simple molecules that are stable at the photospheric temperatures of the cool giants. An indication of the metallicity effect on mass loss can therefore be obtained by studying the molecules in AGB and RSG stars at low metallicity.

AGB stars are classified into two major spectral types: carbon-rich stars and oxygen-rich stars. In photospheric chemistry, $\mathrm{CO}$ molecules are formed first as their bonding energy is highest. In a carbon-rich environment, carbon atoms remaining after $\mathrm{CO}$ formation are incorporated into carbon-bearing molecules, such as $\mathrm{C}_{2}, \mathrm{C}_{2} \mathrm{H}_{2}, \mathrm{CN}$, and $\mathrm{HCN}$. Oxygen-bearing molecules such as $\mathrm{H}_{2} \mathrm{O}, \mathrm{SiO}, \mathrm{CO}_{2}$ are formed in oxygen-rich stars. There is additionally a minor group of stars, S-type stars, which have carbon-to-oxygen ratio ( $\mathrm{C} / \mathrm{O}$ ratio) close to unity, and where $\mathrm{CO}$ molecules are prominent in the infrared spectra.

Previous surveys of AGB stars in the local group galaxies have shown that the ratio of carbon-rich stars to oxygenrich ones depends on the metallicity of the galaxies (e.g. Groenewegen 1999). In the solar neighbourhood, about $90 \%$ of the late AGB stars are oxygen-rich, while in the SMC, which has lower metallicity, about $80 \%$ are carbon-rich. This is because a fixed amount of carbon injected into the envelope has a larger effect for a low metallicity, leading to a higher C/O ratio (Lattanzio \& Wood 2003), but also because dredge-up of newly synthesized carbon atoms is more efficient in AGB stars of lower metallicity (Vassiliadis \& Wood 1993). Marigo (2002) concludes that the carbon-to-oxygen $(\mathrm{C} / \mathrm{O}) \mathrm{ra}-$ tio should be systematically higher in carbon-rich stars at lower metallicity. Spectroscopic evidence of high $\mathrm{C} / \mathrm{O}$ ratio could be found in data of extra-galactic AGB stars. van Loon et al. (1999a) presented the first $L$-band spectra of LMC carbon stars, and found that the equivalent widths of the $3.1 \mu \mathrm{m} \mathrm{HCN}$ and $\mathrm{C}_{2} \mathrm{H}_{2}$ bands appear to be similar in LMC stars and in Galactic stars. Matsuura et al. (2002b, hereafter Paper I) found that the equivalent widths of the $3.1 \mu \mathrm{m}$ band and the $3.8 \mu \mathrm{m} \mathrm{C}_{2} \mathrm{H}_{2}$ band are larger in LMC stars than in Galactic stars. This shows that abundances of carbon-bearing molecules do not scale simply with the metallicity of the host galaxies; instead, carbon synthesized inside AGB stars should result in the higher $\mathrm{C} / \mathrm{O}$ ratio in the LMC stars. The high $\mathrm{C} / \mathrm{O}$ ratio compensates for the low elemental abundances: carbon-bearing molecules are abundant in carbon stars at lower metallicity.

Here we present an observational study of infrared molecular bands in AGB/RSG stars in nearby galaxies. The target galaxies are the Large Magellanic Cloud (LMC) and the Small Magellanic Cloud (SMC), with metallicities of about half and one-quarter of the solar value, respectively, and the Sagittarius Dwarf Spheroidal Galaxy (SgrD), where the two planetary nebulae and the AGB stars have $[\mathrm{Fe} / \mathrm{H}]=-0.55$ (Dudziak et al. 2000); colour-magnitude diagrams show a range of metallicities down to $[\mathrm{Fe} / \mathrm{H}]=-1.15$ (Marconi 1998). The spectra target $\mathrm{HCN}$ and $\mathrm{C}_{2} \mathrm{H}_{2}$ bands from carbon-rich stars and $\mathrm{SiO}$ bands from oxygen-rich stars. We choose these molecules because they are parent molecules for dust grain formation (via PAH and silicate), and their abundance could affect the dust formation efficiency and, therefore, the mass-loss rates of the dust-driven winds of these stars.

\section{Observations}

We selected AGB stars and late-type supergiants in the LMC, the SMC and the SgrD from published catalogues: Trams et al. (1999b); van Loon et al. (1999a) for LMC stars, Groenewegen \& Blommaert (1998) for SMC stars, and Whitelock et al. (1996, 1999) for SgrD stars. The selections are based on $L$-band magnitudes and known classification (oxygen-rich or carbonrich). The observed targets are listed in Table 1. The LMC and the SMC contain luminous and high mass-loss stars that are likely to be young (intermediate-mass) stars. The SgrD stars are older, with a likely age around $5 \mathrm{Gyr}$, and are optical carbon stars without evidence for high mass loss.

$L$-band spectra were observed with ISAAC on the Very Large Telescope (VLT) at ESO Paranal, Chile on 12th-14th of December 2001 (LMC targets) and 24th of July 2002 (SMC and SgrD targets). The sky conditions were clear on December 13th, and thin cloudy on the 12th and 14th of December and on the 24th July. On cloudy nights, especially on July 24th, the sky conditions were variable, and cancellation of atmospheric telluric lines was not ideal. We used chop-andnod to subtract the sky background. Jitter is used along the slit to minimize the influence of hot pixels.

We used two ISAAC instrument modes: low spectral resolution (LR) and medium resolution (MR). The LR mode covers the entire $L$-band, so this mode was used mainly for carbon-rich stars. The MR mode has a spectral coverage of $0.255 \mu \mathrm{m}$, and was used to resolve the $\mathrm{SiO}$ band heads against the continuum in oxygen-rich stars. The wavelength resolutions $(R=\lambda / \Delta \lambda)$ are 360 (1-arcsec slit) and 600 (0.6-arcsec slit) in LR mode, and 2000 (1-arcsec slit) and 3300 (0.6-arcsec slit) in MR mode.

Telluric standards, which were B dwarfs and giants from the Hipparcos catalog, were observed after each target observation. The spectrum of the telluric standard is assumed to be a blackbody with an effective temperature based on the spectral type from the Hipparcos catalog. $\operatorname{Br} \alpha$ and, in some cases, other hydrogen recombination lines and the $\mathrm{He} \mathrm{I}$ line are detected in the telluric standards. We assume a Gaussian profile for these lines. Although Starck broadening will affect the profile, we ignore this effect because its precise calculation requires a hydrostatic model. The cancellation of the atmospheric lines is not good at the $\operatorname{Br} \alpha$ and He I wavelength in some cases. The $3.308-3.320 \mu \mathrm{m}$ region of the spectra is not used in the discussion, because several telluric methane bands are saturated and cannot be removed. The absolute flux is scaled to $L$-band magnitude, which is either taken from Trams et al. (1999b), or estimated from the spectral type, $V$-band magnitude, and colour of the telluric standard; it is uncertain to within a factor of two. The data are reduced using the eclipse package and IDL. An exposure of thermal emission from the twilight sky is used for flat-fielding. The wavelength calibration is based on exposures of an Ar+Xe arc lamp with the same wavelength setting as the target observations. The flux error is estimated from ten nearby sky pixels in the ISAAC CCD image. 


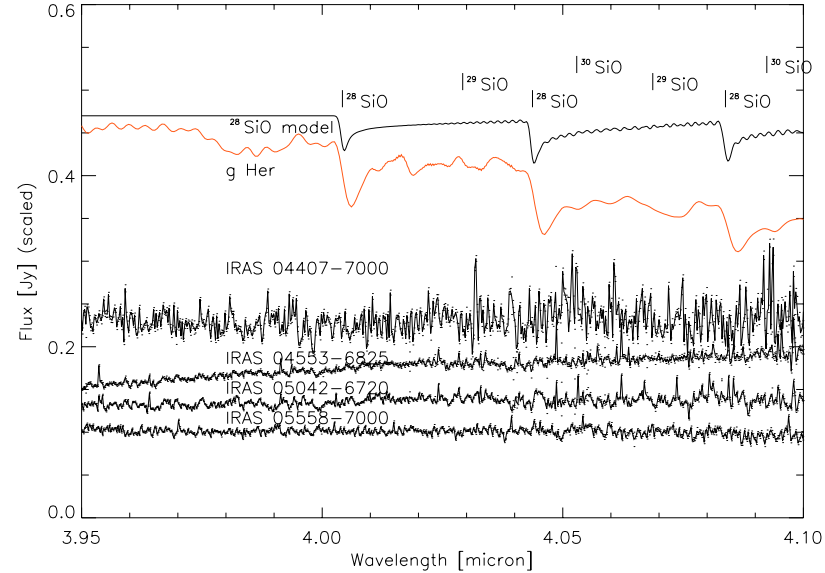

Fig. 1. The 3.85-4.10 $\mu \mathrm{m}$ spectra of four stars LMC oxygen-rich stars. For comparison, a spectrum of the Galactic giant star g Her, and a model spectrum of $\mathrm{SiO}$ bands, are also shown. Wavelength of ${ }^{28} \mathrm{SiO}$, ${ }^{29} \mathrm{SiO},{ }^{30} \mathrm{SiO}$ bands are indicated. $10 \sigma$ errors are plotted as dots.

We re-analysed the data for the LMC carbon stars whose equivalent widths were published in Matsuura et al. (2002b). Only the positive images of chop-and-nod were used in Paper I, while in this paper both the positive and negative images are used.

\section{Spectra of oxygen-rich stars}

The 3.95-4.10 $\mu \mathrm{m}$ spectra of four oxygen-rich stars in the LMC are shown in Fig. 1, where a model spectrum of the $\mathrm{SiO}$ bands and a spectrum of a Galactic $\mathrm{M}$ giant star ( $\mathrm{g}$ Her) are plotted as references. The data of $\mathrm{gHer}$ were taken with ISO/SWS AOT S6 (resolution of about 1600-2000), and the spectrum of g Her clearly shows $\mathrm{SiO}$ bands. Parameters for the model spectra of $\mathrm{SiO}$ are taken from Langhoff \& Bauschlicher (1993) and Sauval \& Tatum (1984).

The oxygen-rich stars in the LMC show no clear features in their spectra. Although the observations targeted the $\mathrm{SiO}$ bands, no $\mathrm{SiO}$ bands are detected in any of these stars.

The quality of the IRAS $04407-7000$ spectrum is poor. A bump at $4.05 \mu \mathrm{m}$ is caused by insufficient cancellation of atmospheric lines due to $\operatorname{Br} \alpha$ absorption in the telluric standard star, and does not imply a $\operatorname{Br} \alpha$ emission line.

A spectrum covering the full $L$-band of the oxygen-rich star IRAS 05148-6730 is shown in Fig. 2. A spectrum of g Her and model spectra of $\mathrm{OH}$ and $\mathrm{SiO}$ lines are also plotted. Parameters for the model spectra of $\mathrm{OH}$ lines are taken from HITRAN (Rothman et al. 1998). The ISO/SWS spectrum of $\mathrm{g}$ Her shows $\mathrm{OH}, \mathrm{SiO}$ and possibly weak $\mathrm{H}_{2} \mathrm{O}$ bands shortwards $3.2 \mu \mathrm{m}$. In the spectrum of IRAS 05148-6730 the decline of the flux shortward of $3.0 \mu \mathrm{m}$ could be due to hightemperature $\mathrm{H}_{2} \mathrm{O}$ bands; other than this, no particular molecular bands are detected. Most of the features are residual atmospheric lines and noise, rather than $\mathrm{OH}$ or $\mathrm{SiO}$ bands. The $\mathrm{H}_{2} \mathrm{O}$ continuous absorption changes its shape depending on the excitation temperature and column density. The $\mathrm{H}_{2} \mathrm{O}$ feature in IRAS 05148-6730 will not necessarily resemble the one in $\mathrm{g}$ Her.

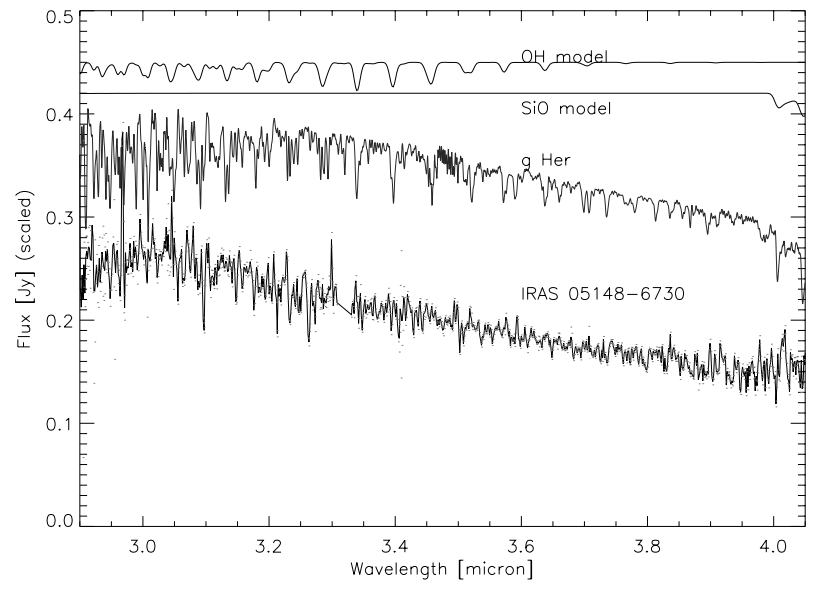

Fig. 2. The spectrum of one of the oxygen-rich stars, IRAS 05148-6730, which covers the entire $L$-band. For comparison, a spectra of $\mathrm{gHer}$, and $\mathrm{OH}$ and $\mathrm{SiO}$ models are also plotted. $10 \sigma$ errors are plotted as dots.

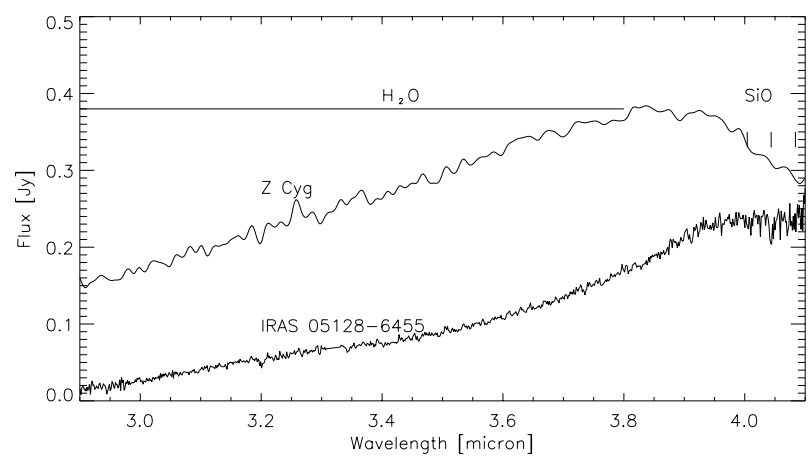

Fig. 3. The spectrum of IRAS 05128-6455, compared the spectrum of the galactic oxygen-rich Mira, Z Cyg $(R \sim 300$; Matsuura et al. $2002 \mathrm{a}$ ), which shows $\mathrm{H}_{2} \mathrm{O}$ and $\mathrm{SiO}$ bands. Deep continuous absorption shortwards of $3.8 \mu \mathrm{m}$ in IRAS $05128-6455$ is due to $\mathrm{H}_{2} \mathrm{O}$. SiO bands may be detected, although the band strength is almost the same as the noise level. A small dip at $3.2 \mu \mathrm{m}$ is the $\mathrm{OH} Q$-band.

\subsection{IRAS 05128-6455}

Figure 3 shows the spectra of IRAS 05128-6455 and a comparison galactic oxygen-rich star Z Cyg. Z Cyg shows continuous $\mathrm{H}_{2} \mathrm{O}$ absorption up to $3.8 \mu \mathrm{m}$, which is also seen in IRAS 05128-6455. SiO bands are found in IRAS 05128-6455 spectra, although the intensity is almost at noise level, due to insufficient cancellation of atmospheric lines. A small dip at $3.20 \mu \mathrm{m}$ found both in Z Cyg and in IRAS 05128-6455 is part of the $\mathrm{H}_{2} \mathrm{O}$ feature with $\mathrm{OH}$ blending (also found in $\mathrm{g}$ Her and the $\mathrm{OH}$ model spectrum in Fig. 2).

IRAS 05128-6455 was misclassified as a carbon star in Trams et al. (1999b), based on the infrared colours and on the ISO/PHOT spectrum. The latter suggests some absorption-like structure at $3 \mu \mathrm{m}$, which could be the $\mathrm{HCN}$ and $\mathrm{C}_{2} \mathrm{H}_{2}$ bands. However, we could not find such an absorption feature in the ISAAC/VLT spectra. The structure in the ISO/PHOT spectrum appears to be the result of an inaccuracy in the ISO/PHOT detector response curve at the time of reduction. Such an anomaly is also found in the ISO/PHOT spectrum of the oxygen-rich star WOH G64 (Trams et al. 1999b). IRAS 05128-6455 is clearly 
Table 1. Targets and log of the ISAAC observations. C/O classification shows the basis of classification into oxygen-rich or carbon-rich (cand: candidate) in publications. Opt sp: optical spectra, IR sp: infrared spectra (mainly molecule features), dust: $9.8 \mu \mathrm{m}$ silicate band. $t_{\exp }$ is the total exposure time in minutes. Observing mode shows either LR or MR and the slit width in arcsec. Observing dates are 12th and 14th December, 2001 for LMC stars, and 24th July 2002 for the SMC and SgrD. Ref: reference for the coordinates.

\begin{tabular}{|c|c|c|c|c|c|c|c|}
\hline Name & Alternative name & $\begin{array}{l}\text { Coordinates } \\
(J=2000)\end{array}$ & $\mathrm{C} / \mathrm{O}$ classification & $t_{\exp }$ & $\begin{array}{l}\text { LR/MR } \\
+ \text { Slit }\end{array}$ & $\begin{array}{l}\text { Telluric } \\
\text { standard }\end{array}$ & Ref. \\
\hline \multicolumn{8}{|l|}{$L M C$} \\
\hline \multicolumn{8}{|l|}{ Oxygen-rich } \\
\hline IRAS 04407-7000 & LI-LMC 4 & $044028.4-695513$ & $\begin{array}{l}\text { dust }^{10}, \text { Maser }^{6,14} \\
\text { M7. }\end{array}$ & 60 & MR 0.6 & HIP 017543 & 9,10 \\
\hline IRAS 04553-6825 & WOH SG 64 & $045510.1-682035$ & $\begin{array}{l}\text { M7.5: Opt } \mathrm{sp}^{1} \\
\text { supergiant }^{1,16} \\
\text { Maser }^{6,12,13,14,15,21}\end{array}$ & 33 & MR 0.6 & HIP 027534 & 10,17 \\
\hline IRAS $05042-6720$ & HV 888 & $050414.3-671617$ & $\begin{array}{l}\text { Opt sp M1I }{ }^{16}, \text { M4Ia }^{7} \text {, } \\
\text { supergiant }^{8,16}\end{array}$ & 33 & MR 1.0 & HIP 027534 & 10 \\
\hline IRAS $05148-6730$ & LI-LMC 663 & $051449.9-672719$ & $\begin{array}{l}\text { Opt Sp M1I }{ }^{16} \\
\text { supergiant }^{8,16}\end{array}$ & 66 & LR 0.6 & HIP 025889 & 10 \\
\hline IRAS 05558-7000 & LI-LMC 1790 & $055520.8-700005$ & dust $^{10}$, Maser $^{6}$ & 63 & MR 0.6 & HIP 029134 & 9,10 \\
\hline \multicolumn{8}{|c|}{ Oxygen-rich (misclassified as carbon-rich before) } \\
\hline IRAS 05128-6455 & LI-LMC 1880 & $051304.6-645140$ & $\begin{array}{l}\text { carbon:IR colour, } \\
\text { IR sp }{ }^{10}\end{array}$ & 63 & LR 0.6 & HIP 027534 & 10 \\
\hline \multicolumn{8}{|l|}{ Carbon-rich } \\
\hline IRAS 04286-6937 & LI-LMC 1825 & $042830.3-693049$ & IR colour $^{10}$ & 30 & LR 0.6 & HIP 021949 & 9,10 \\
\hline IRAS 04496-6958 & LI-LMC 57 & $044918.6-695314$ & $\begin{array}{l}\text { Opt sp } \\
\text { Silicate carbon } \operatorname{star}^{10}\end{array}$ & 66 & LR 0.6 & HIP 029635 & 9,10 \\
\hline IRAS 04539-6821 & LI-LMC 141 & $045346.3-681612$ & IR colour $^{10}$ & 63 & LR 0.6 & HIP 021949 & 9,10 \\
\hline IRAS 04557-6753 & LI-LMC 198 & $045538.9-674910$ & IR colour $^{10}$ & 66 & LR 0.6 & HIP 016368 & 9,10 \\
\hline IRAS 05112-6755 & LI-LMC 570 & $051110.1-675217$ & IR sp/IR colour ${ }^{10}$ & 66 & LR 0.6 & HIP 027534 & 9,10 \\
\hline $46.3-675547^{10}$ & Opt sp? & & & & & & \\
\hline SHV 0521050-690415 & & $052046.8-690125$ & Opt $\mathrm{sp}^{3}$, IR colour ${ }^{10}$ & 66 & LR 0.6 & HIP 025889 & 3,10 \\
\hline IRAS 06025-6712 & LI-LMC 1813 & $060231.0-671247$ & IR $\mathrm{sp}^{11}$ & 36 & LR 0.6 & HIP 029727 & 9 \\
\hline \multicolumn{8}{|l|}{ SMC carbon-rich } \\
\hline SMC-S2 & & $003659.6-741950$ & $\begin{array}{l}\text { Opt sp }{ }^{16} \\
\text { Post-AGB cand. }{ }^{2,18}\end{array}$ & 48 & LR 0.6 & HIP 012389 & 2 \\
\hline SMC-S30 & & 0122 29.3-71 0938 & Opt sp ${ }^{2}$ & 60 & LR 0.6 & HIP 012389 & 2 \\
\hline \multicolumn{8}{|l|}{ SgrD carbon-rich } \\
\hline Sgr-C1 & & $184624.0-301509$ & Opt $\mathrm{sp}^{5}$ & 60 & LR 0.6 & HIP 093542 & 19 \\
\hline Sgr-C3 & & $185250.4-295631$ & Opt sp 5 & 32 & LR 0.6 & HIP 093542 & 19 \\
\hline Sgr-UKST3 & & $184639.1-304552$ & Opt $\mathrm{sp}^{20}$ & 60 & LR 0.6 & HIP 093542 & 19 \\
\hline Sgr-UKST15 & & $185655.9-312440$ & Opt $\mathrm{sp}^{20}$ & 60 & LR 0.6 & HIP 093542 & 19 \\
\hline
\end{tabular}

1: Elias et al. (1986); 2: Groenewegen \& Blommaert (1998); 3: SHV: Hughes (1989); 4: Hughes (1990); 5: Ibata et al. (1995); 6: Marshall et al. (2004); 7: HV: Payne-Gaposchkin (1971); 8: Reid et al. (1990); 9: LI-LMC: Schwering \& Israel (1990); 10: Trams et al. (1999b); 11: van Loon et al. (2003); 12: van Loon et al. (1996); 13: van Loon et al. (1998a); 14: van Loon et al. (1998b); 15: van Loon et al. (2001); 16: van Loon (preparation); 17: WOH: Westerlund et al. (1981); 18: Whitelock et al. (1989); 19: Whitelock et al. (1996); 20: Whitelock et al. (1999); 21: Wood et al. (1992).

an oxygen-rich star, as shown by its optical spectrum (van Loon et al. in preparation). The spectral type is M9 (van Loon et al.)

\subsection{Equivalent width of the SiO bands}

The equivalent width $(E W)$ of the $\mathrm{SiO}$ bands $\left(E W_{\mathrm{SiO}}\right)$ are measured for LMC oxygen-rich stars and compared with the galactic sample (Fig. 4, Table 3). The definition of $E W_{\mathrm{SiO}}$ is similar to the one in Aringer et al. (1997, 1999), who studied $\mathrm{SiO}$ bands in Galactic AGB stars. The $E W_{\mathrm{SiO}}$ is the sum of equivalent widths of three strong ${ }^{28} \mathrm{SiO}$ bands. The wavelengths used for the continuum and the $\mathrm{SiO}$ bands are the same as Aringer et al. (1997, 1999). In Aringer et al. (1997), the continuum is estimated by a polynomial fit. Here we use a linear fit, because third-order polynomials were inappropriate and because we occasionally have insufficient data to attempt higher order fits. This difference of fitting has little influence on the measured $E W$, as tested on SWS spectra: less than $5 \AA$. 


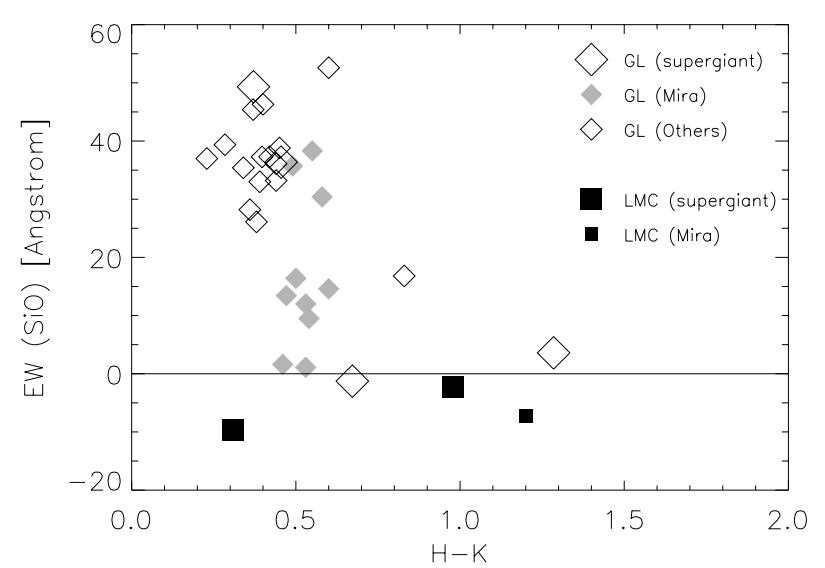

Fig. 4. The equivalent width of the $\mathrm{SiO}$ bands. For Galactic stars, supergiants and Mira variables sources are shown by different symbols. Semi-regular variables and unknown variable types are plotted as "others".

The exception is KK Per, for which we found a difference of about $10 \AA$. The precise wavelength regions used for the continuum are summarized in Table 2 .

The one- $\sigma$ error in the $E W$ is propagated from the noise in the spectrum, and is less than $1 \AA$. However, in practice, the error of the local continuum estimate has a larger uncertainty, as mentioned above. Aringer et al. (1999) found a similar uncertainty up to $5 \AA$.

To allow comparison with the LMC sample, we used the $\mathrm{SiO} E W \mathrm{~s}$ of Galactic AGB stars given in Aringer et al. (1999). In addition, we reduced ISO/SWS spectra that were observed in AOT 6 (wavelength resolution of about $R=1600-2000$ ), and AOT 1 speed $4(R=1000-1400)$ which was used for $\alpha$ Ori. We also use reduced ISO/SWS AOT 6 spectra from Sloan et al. (2003).

The measured $E W$ of $\mathrm{SiO}$ bands are summarized in Table 3. No evidence for absorption bands is found, consistent with a non-detection of SiO. Three LMC stars are affected by data problems and are listed separately; for IRAS 05128-6455 and IRAS 05148-6730 the wavelength resolution is not high enough to separate the continuum and the absorption, while IRAS 04407-7000 has a poor quality spectrum.

Figure 4 shows the $E W_{\mathrm{SiO}}$ of LMC and Galactic oxygenrich stars, as a function of $H-K$ colour. Here we plot the $E W_{\mathrm{SiO}}$ of three LMC stars only, avoiding any with poor quality data. The $H-K$ colour is the measure of the effective temperature for blue stars, but not for red stars where the colour is strongly affected by dust. For both LMC and Galactic stars, large $E W_{\mathrm{SiO}}$ stars (larger than $20 \AA$ ) are absent among red stars $(H-K>0.7)$. Within our sample of blue Galactic stars, there is no difference in $E W_{\mathrm{SiO}}$ between supergiants and giants (Mira and semi-regular variables) as is consistent with previous observations (Wallace \& Hinkle 2002; Heras et al. 2002).

\section{Spectra of carbon-rich stars}

Figure 5 shows the ISAAC spectra of carbon-rich stars in the LMC, while spectra of carbon-rich AGB stars in the SMC and SgrD are shown in Fig. 6. Bands of different molecules are
Table 2. The definition of equivalent width $(E W)$ for the molecular absorption bands. The continuum level is linearly interpolated from the wavelength region in the last column. $\mathrm{SiO}$ molecules show three strong bands from $4.0-4.1 \mu \mathrm{m}$, and $E W_{\mathrm{SiO}}$ is the total of these three bands.

\begin{tabular}{llll}
\hline \hline Name & Molecules & $\begin{array}{l}\text { Measured } \\
\text { region }[\mu \mathrm{m}]\end{array}$ & $\begin{array}{l}\text { Continuum } \\
{[\mu \mathrm{m}]}\end{array}$ \\
\hline$E W_{3.1}$ & $\mathrm{HCN}, \mathrm{C}_{2} \mathrm{H}_{2}$ & $2.97-3.35$ & $2.94-2.97,3.35-3.40$ \\
$E W_{3.5}$ & $\mathrm{HCN}$ & $3.56-3.58$ & $3.51-3.56,3.58-3.63$ \\
$E W_{3.8}$ & $\mathrm{C}_{2} \mathrm{H}_{2}$ & $3.60-4.00$ & $3.50-3.60,4.00-4.10$ \\
\hline$E W_{\mathrm{SiO}}$ & $\mathrm{SiO}$ & $4.0025-4.0200$ & $3.95-3.96,3.99-4.00$ \\
& & $4.0425-4.0600$ & $4.036-4.04$ \\
& & $4.0825-4.1000$ & $4.076-4.080$ \\
\hline
\end{tabular}

found in the $L$-band: the $3.1 \mu \mathrm{m} \mathrm{HCN}+\mathrm{C}_{2} \mathrm{H}_{2}$ band, 3.4 and $3.7 \mu \mathrm{m} \mathrm{CH}, 3.5 \mu \mathrm{m} \mathrm{HCN}$, and $3.8 \mu \mathrm{m} \mathrm{C}_{2} \mathrm{H}_{2}$. The last identification is probable rather than certain.

The $3.1 \mu \mathrm{m} \mathrm{HCN}+\mathrm{C}_{2} \mathrm{H}_{2}$ is commonly seen in carbonrich stars, but is more pronounced in bluer stars, such as IRAS 04496-6958. The sharp absorption at $3.57 \mu \mathrm{m}$ is due to HCN Q-branch lines. The obvious features are blend of lines from $\left(00^{0} 0\right)$ to $\left(01^{1} 1\right)$ and some other transitions (Harris et al. 2003). Lines with other transitions of the HCN Q-branch, around $3.5 \mu \mathrm{m}$, are not obvious in our spectra. The $3.5 \mu \mathrm{m}$ feature is found in only a few of the extra-galactic stars: IRAS 04496-6958, IRAS 06025-6712, SMC S30, and probably IRAS 04557-6753 and IRAS 05112-6755.

A non-detection of the $3.5 \mu \mathrm{m}$ HCN band does not immediately mean no contribution by $\mathrm{HCN}$ to the $3.1 \mu \mathrm{m}$ band. The absorbance ratio of the $3.1 \mu \mathrm{m} / 3.5 \mu \mathrm{m} \mathrm{HCN}$ bands is approximately 100 (Harris et al. 2002), and the $3.1 \mu \mathrm{m}$ feature is easily formed even at lower $\mathrm{HCN}$ column density.

The identification of the $\mathrm{C}_{2} \mathrm{H}_{2}$ feature is still tentative. Goebel et al. (1981) noted the $3.8 \mu \mathrm{m}$ feature on a KAO spectrum of the carbon star V CrB. Hron et al. (1998)'s model calculation shows a $\mathrm{C}_{2} \mathrm{H}_{2}$ band at $3.8 \mu \mathrm{m}$, and they identify this with a feature in the ISO/SWS spectra of the Galactic carbon star R Scl. However, the absorption in the R Scl spectrum starts only at $3.9 \mu \mathrm{m}$, which could indicate CS (Aoki et al. 1998). The identification of the $3.8 \mu \mathrm{m}$ band, which stretches from 3.6 to $4.0 \mu \mathrm{m}$, as a $\mathrm{C}_{2} \mathrm{H}_{2}$ feature is still not fully fixed.

There are several other weak lines visible in the spectra around $3.4 \mu \mathrm{m}$ and at $3.7 \mu \mathrm{m}$, where weak lines are seen superposed on the broad $\mathrm{C}_{2} \mathrm{H}_{2}$ band. These lines are due to $\mathrm{CH}$ (Fig. 7), whose bands are found in the Galactic carbon star TX Psc (Ridgway et al. 1984; Aoki et al. 1998): its ISO/SWS spectrum (Aoki et al. 1998) is added in Fig. 7 for comparison. The resolution of the TX Psc spectrum is $R \sim 2000$, higher than the ISAAC LR spectra $(R=600)$, and the $\mathrm{CH}$ bands appear more clearly. In the ISAAC spectra, the $\mathrm{CH} v=1-0$, and $v=3-2$ bands are easy to find while $v=2-1$ at $3.5 \mu \mathrm{m}$ is not obvious. This could be because of the blend with the $3.5 \mu \mathrm{m} \mathrm{HCN}$ bands. Note that $\mathrm{C}_{2} \mathrm{H}_{2}$, which contains $\mathrm{C}-\mathrm{H}$ bonds, may also contribute narrow features at $3.8 \mu \mathrm{m}$, but these are not definitely detected. 
Table 3. The equivalent width of $\mathrm{SiO}$ bands (unit is $\AA$ ). The date is that of either ISAAC or ISO/SWS observations in $d d m m y y$ format, which is different from the observing date of the magnitudes. Column four $\left(\phi_{\mathrm{K}}\right)$ lists the pulsation phase during the SiO observations, which is estimated from infrared $K$-band light curve (Whitelock et al. 2003), where 0.0 and 1.0 are the maxima. Uncertainty should be about 0.1 . The optical phase $\left(\phi_{\text {opt }}\right)$ is estimated for the ISO sample, based on the AAVSO light curve. The optical phase is usually about $0.1-0.2$ of a cycle ahead of the infrared phase. Photometric magnitudes are taken from Trams et al. (1999b) for the LMC sample, and 2MASS for the Galactic sample. Luminosity class and spectral types for Galactic stars are taken from Simbad.

\begin{tabular}{|c|c|c|c|c|c|c|c|}
\hline Name & Sp. type & Date & $\phi_{\mathrm{K}}$ & $\phi_{\mathrm{opt}}$ & $E W_{\mathrm{SiO}}$ & $H$ & $K$ \\
\hline \multicolumn{8}{|l|}{ LMC sample } \\
\hline IRAS $04553-6825$ & M7.5, supergiant & $12-12-01$ & & & -2.2 & 7.96 & 6.98 \\
\hline IRAS 05042-6720 & M1I, M4Ia, supergiant & $12-12-01$ & & & -9.7 & 7.20 & 6.89 \\
\hline IRAS 05558-7000 & & $13-12-01$ & 0.87 & & -7.3 & 10.10 & 8.90 \\
\hline \multicolumn{8}{|c|}{ LMC sample (Poorer quality in $E W_{\mathrm{SiO}}$ ) } \\
\hline IRAS 04407-7000 & & $14-12-01$ & 0.82 & & -24.2 & 8.92 & 8.18 \\
\hline IRAS 05128-6455 & & $13-12-01$ & & & 6.0 & 12.10 & 10.55 \\
\hline IRAS 05148-6730 & M1I, supergiant & $15-12-01$ & & & 17.5 & & \\
\hline \multicolumn{8}{|c|}{ Galactic sample from ISO } \\
\hline R Aql & Mira & $19-03-97$ & & 0.25 & 13.4 & $-0.355 \pm 0.218$ & $-0.826 \pm 0.230$ \\
\hline VY CMa & M5Ia,e & 21-11-97 & & & 3.6 & $1.576 \pm 0.210$ & $0.291 \pm 0.236$ \\
\hline g Her & M6III, SR & $23-08-96$ & & & 39.4 & $-1.850 \pm 0.138$ & $-2.134 \pm 0.158$ \\
\hline$\alpha$ Ori & M1Iab, SR & 08-10-97 & & & 49.3 & $-4.007 \pm 0.162$ & $-4.378 \pm 0.186$ \\
\hline$\rho$ Per & M4II, SR & 05-03-98 & & & 37.0 & $-1.675 \pm 0.158$ & $-1.904 \pm 0.152$ \\
\hline KK Per & M2Iab-Ib & 03-02-98 & & & 36.4 & $2.138 \pm 0.166$ & $1.683 \pm 0.206$ \\
\hline VX Sgr & M5/M6III, M4eIa, SR & $13-10-96$ & & & -1.3 & $0.550 \pm 0.304$ & $-0.122 \pm 0.362$ \\
\hline SW Vir & M7III, SR & $20-07-96$ & & & 37.3 & $-1.606 \pm 0.260$ & $-2.003 \pm 0.336$ \\
\hline
\end{tabular}

Sgr-C1 shows a flat spectrum without any feature in the $L$-band: no bands are visible. The flat spectrum (in Jy) at $L$ implies a red colour which shows that this is a late-type star, but we were unable to confirm that it is actually a carbon star. Sgr-C1 is one of the first stars found in SgrD (Ibata et al. 1995), and it is an optically classified carbon star. It is unlikely that we took the spectrum of the wrong object, because the VLT pointing is in general accurate enough and the acquisition image of ISAAC is consistent with the finding chart in Whitelock et al. (1996). Whitelock et al. (1999) show that the $K$-band magnitude of Sgr-C1 is $11.2 \mathrm{mag}$, fainter than the three other Sgr-D carbon-rich stars (9.4, 9.8 and $9.7 \mathrm{mag})$. The luminosity of Sgr-C1 is $M_{\mathrm{K}} \sim-6.1 \mathrm{mag}$, which is not near the tip of the AGB sequence (Whitelock et al. 1996). All these suggest that Sgr-C1 could be an extrinsic carbon star.

\subsection{The post-AGB star, SMC-S2}

In the spectrum of SMC-S2, the $3.3 \mu \mathrm{m}$ PAH band is detected (Fig. 8). Although this confirms its carbon-rich nature, it also shows that this object is unlikely to be an AGB star, as $\mathrm{PAH}$ emission is never seen in AGB stars. The temperatures of AGB stars are too low to excite PAHs: this requires UV or optical photons. PAH emission is usually seen once the stars reach a temperature of $\sim 5000 \mathrm{~K}$. The presence of $\mathrm{PAH}$ emission indicates that SMC-S2 is a post-AGB star, which was already suspected from its infrared colours and the optical spectra (Whitelock et al. 1989; Groenewegen \& Blommaert 1998). This is probably the first detection of a PAH band in an SMC post-AGB star.
The optical spectrum suggests a K-type star. This is rather cool for PAH excitation but the detected feature is weak. It is possible that the underlying star is hotter than implied by the spectral type and that the spectrum is masked by the stellar envelope. Whitelock et al. (1989) discuss whether the object may be an interacting binary. This cannot be excluded, but the weak variability and high luminosity are consistent with a post-AGB nature, and there is no evidence for ionization in the spectrum.

PAH emission is widespread in the interstellar medium (ISM) of the SMC, as shown by ISOCAM observations (Bot et al. 2004), but at a low abundance relative to the gas as expected from its metallicity. The extinction curve in the bar of the SMC rises very steeply to the UV (Gordon et al. 2003), which can be attributed to PAHs. It is not known whether the PAHs form in the ISM or derive from the same sources as the dust. PAH detection in SMC-S2 shows that PAHs can form in the metal-poor stars, leaving evolved stars as a potential source.

\subsection{Equivalent widths of the molecular bands}

We measured the $E W$ s of the three main molecular bands of carbon-rich stars. The parameters used to measure the $E W$ are summarized in Table 2. The $E W$ s are plotted against infrared colour in Figs. 9-11, where the infrared colour $H-K$ is used as a measure for the effective temperature. The circumstellar excess also affects this colour for redder carbon-rich stars.

For comparison with Galactic stars, we reduced ISO/SWS spectra of carbon stars in the solar neighbourhood, and also used the reduced ISO/SWS spectra of Sloan et al. (2003). The infrared colours for the Galactic stars were taken from the literature (Table 4). 

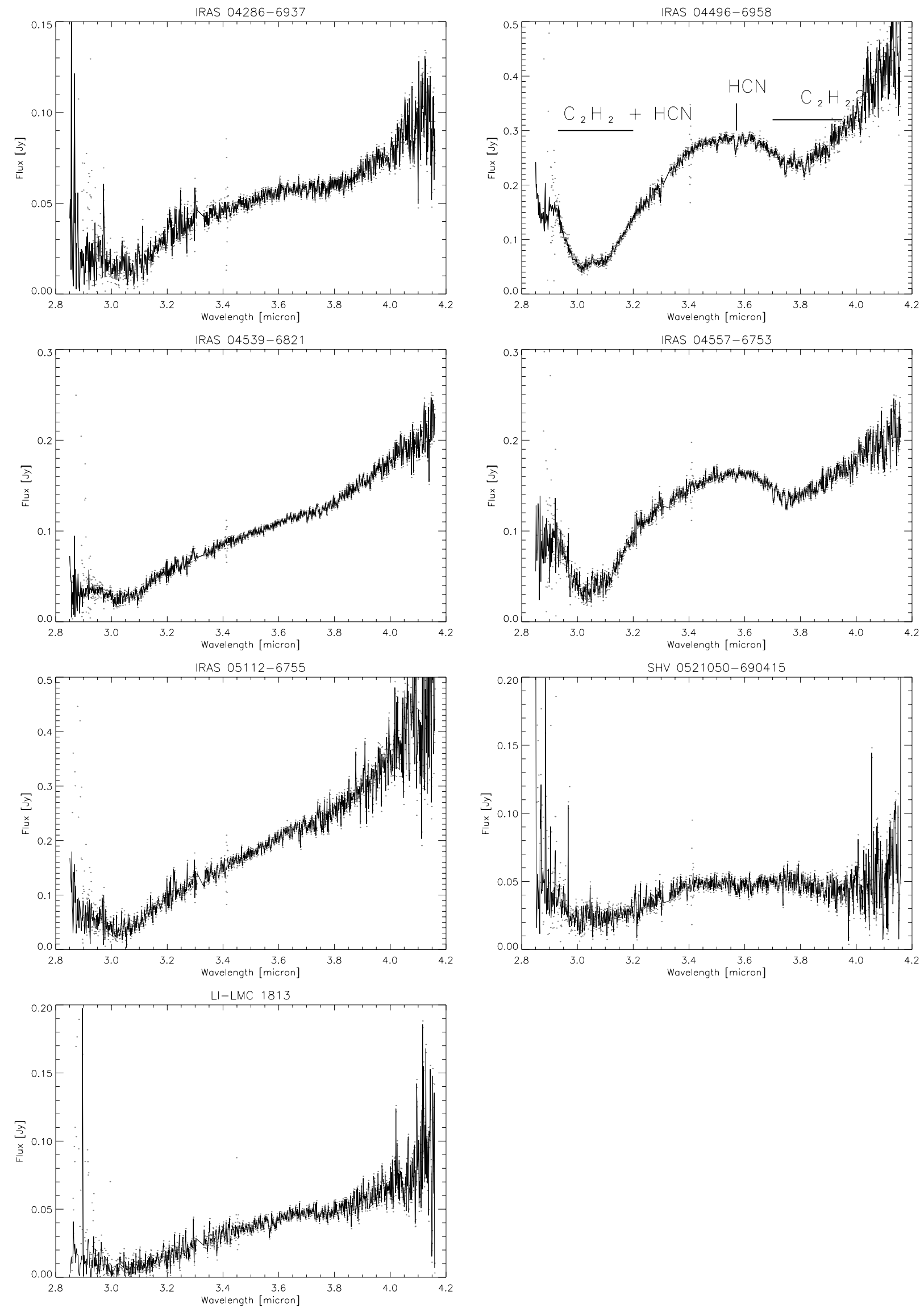

Fig. 5. ISAAC spectra of carbon-rich AGB stars in LMC. Molecule bands, in particular the $3.1 \mu \mathrm{m} \mathrm{C}_{2} \mathrm{H}_{2}+\mathrm{HCN}, 3.5 \mu \mathrm{m} \mathrm{HCN}$, and $3.8 \mu \mathrm{m}$, probably $\mathrm{C}_{2} \mathrm{H}_{2}$ bands, are indicated. $10 \sigma$ errors are plotted as dots. 

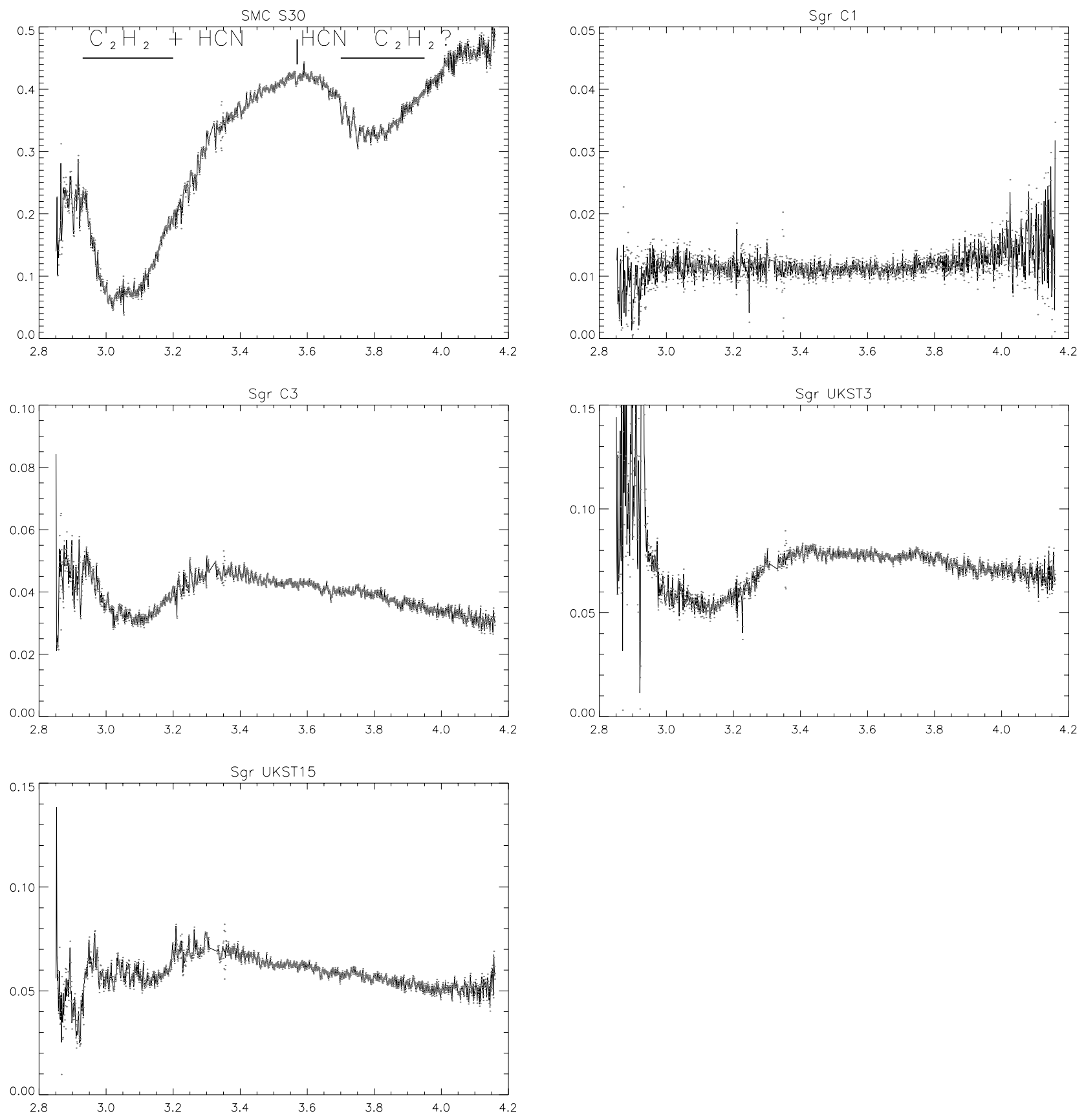

Fig. 6. Same as Fig. 5, but for carbon-rich stars in SMC and SgrD.

As seen in the spectra, the $3.5 \mu \mathrm{m} E W$ is almost negligible in extra-galactic AGB stars, in contrast to the nearby AGB stars which have $E W$ s up to $12 \AA$. Only a few LMC stars show $E W_{3.5}$ larger than $5 \AA$, but in those LMC stars that show the band, this molecular band is as strong as in the Galactic stars. There is a clear tendency for the $E W$ to decrease with increasing $H-K$, but there is also a group of stars at blue $H-K$ colour with very weak HCN bands.

T Dra, V CrB and V460 Cyg were observed with ISO/SWS at several phases. We find evidence for a large time variation in the $\mathrm{HCN} E W_{3.5}$; e.g., V CrB shows variation from 2-10

The $3.8 \mu \mathrm{m} E W$, which probably measures the $\mathrm{C}_{2} \mathrm{H}_{2}$ abundance, is high in red carbon stars $(H-K>1.2)$, as shown in

Fig. 10. This holds true for both the LMC stars and the single SMC star. The SgrD stars do not show the band but this is consistent with their blue colour. Among the Galactic stars, only a few objects show a $3.8 \mu \mathrm{m} \mathrm{C}_{2} \mathrm{H}_{2}$ band.

The $3.1 \mu \mathrm{m}$ band is a mix of $\mathrm{HCN}$ and $\mathrm{C}_{2} \mathrm{H}_{2}$ bands. $E W_{3.1}$ is large in the spectra of the extra-galactic, red carbon stars. The weakness of the $3.5 \mu \mathrm{m}$ band in extra-galactic AGB stars suggests a lower contribution from $\mathrm{HCN}$ in extra-galactic stars than in galactic stars: the $3.1 \mu \mathrm{m}$ band of the extra-galactic stars may have a larger contribution of $\mathrm{C}_{2} \mathrm{H}_{2}$ than Galactic stars.

Both $E W_{3.5}$ and $E W_{3.8}$ decrease towards redder infrared colours; $E W_{3.1}$ increases until $H-K=1.2$, and then $E W_{3.1}$ 


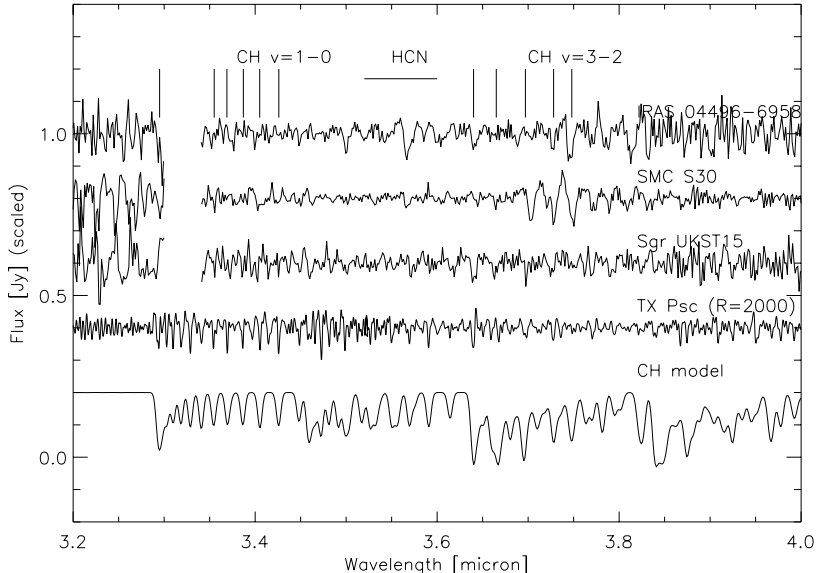

Fig. 7. The flattened spectra of the carbon-rich stars, compared with the $\mathrm{CH}$ model spectrum and with the ISO/SWS spectrum of TX Psc. The CH line list is taken from Jørgensen et al. (1996). The relative intensities of the $\mathrm{CH}$ lines in the model are not always comparable to the observed ones, especially around 3.5 and $3.8 \mu \mathrm{m}$, because of other opacity sources such as the $\mathrm{HCN}$ and $\mathrm{C}_{2} \mathrm{H}_{2}$ bands.

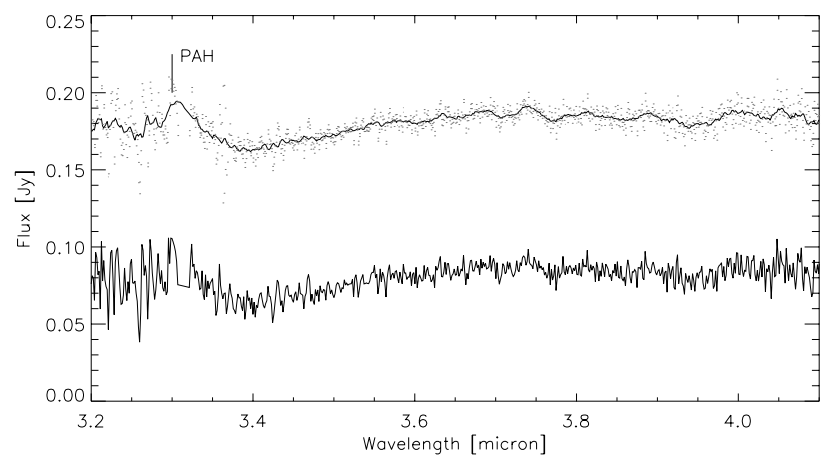

Fig. 8. The spectrum of SMC-S2, and smoothed spectrum to 1/10th of the original wavelength resolution. The $3.3 \mu \mathrm{m}$ PAH band is detected. (The 3.308-3.320 $\mu \mathrm{m}$ region is removed because of strong telluric lines.) $10 \sigma$ errors are plotted as dots.

begins to decreases. This colour corresponds roughly to the tip of the AGB colour sequence: redder stars are affected by circumstellar extinction and/or dust emission. The decline towards red colours suggests that circumstellar emission fills in the absorption features.

\section{Discussion}

\subsection{SiO abundance in extra-galactic oxygen-rich AGB stars}

The SiO bonding energy is one of the highest (after $\mathrm{CO}$ ) amongst the major photospheric molecules. It is therefore not surprising that $\mathrm{SiO}$ bands are commonly detected in latetype stars in our Galaxy, including post-AGB stars (Käufl \& Stangellini 1992) and S-type stars (Wallace \& Hinkle 2002). However, we only found one possible detection of $\mathrm{SiO}$ bands among 6 LMC oxygen-rich stars (three of them are M-type supergiants). This difference needs to be explained.

The LMC oxygen-rich stars are very luminous (van Loon 2000), and three of our targets are supergiants. Possibly

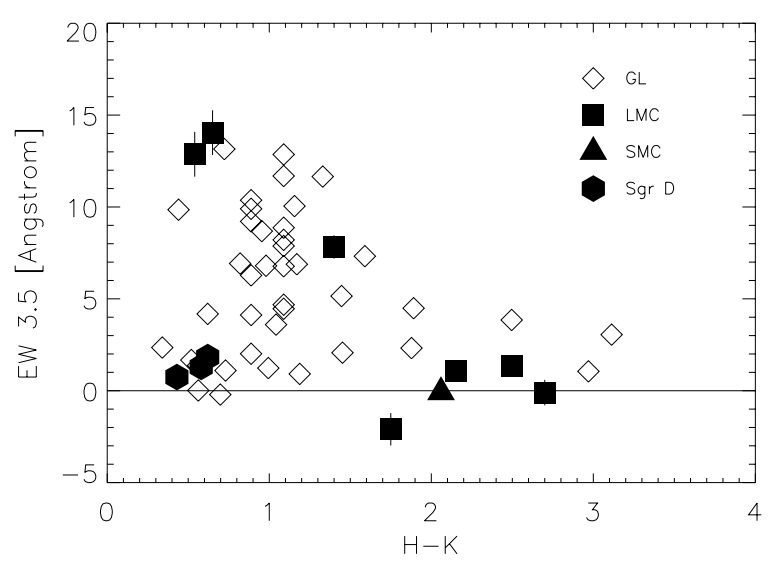

Fig. 9. The HCN equivalent widths of $E W_{3.5}$. The symbols show the host galaxies of the samples.

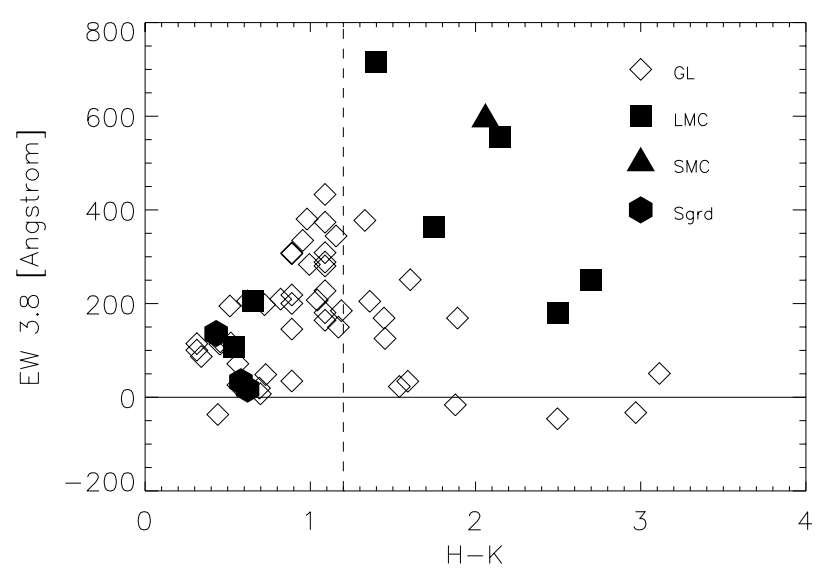

Fig. 10. The $\mathrm{C}_{2} \mathrm{H}_{2}$ equivalent widths of $E W_{3.8}$.

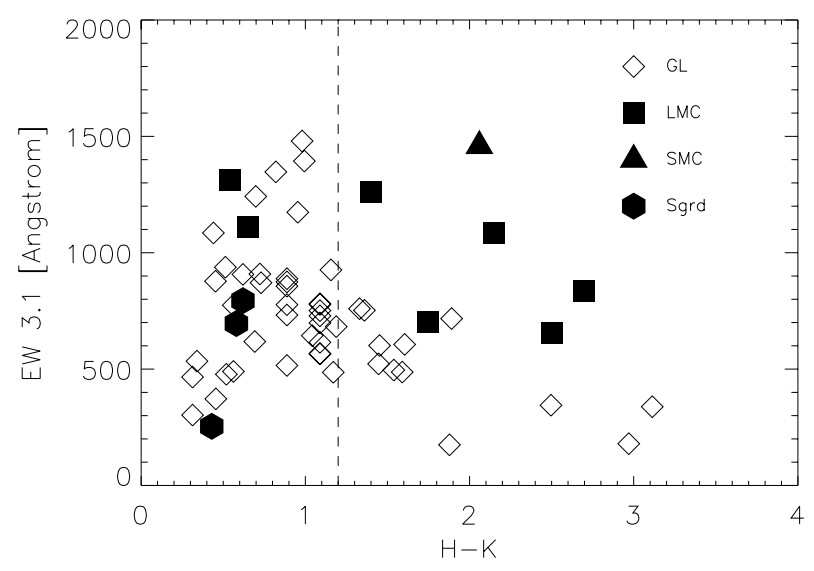

Fig. 11. The $\mathrm{HCN}$ and $\mathrm{C}_{2} \mathrm{H}_{2}$ equivalent widths of $E W_{3.1}$.

they are more luminous than the Galactic comparison stars. However, the luminosity class does not affect the $E W$ of the SiO bands, as shown by Wallace \& Hinkle (2002) and Heras et al. (2002).

The abundance of $\mathrm{SiO}$ is determined by the least abundant component which will generally be silicon. As an $\alpha$-element silicon is not synthesised in AGB stars and its abundance is determined by the composition of the progenitor star at its formation. The major effect on $\mathrm{SiO}$ abundance should be the $\mathrm{Si}$ elemental abundance. The $\mathrm{SiO}$ abundance is limited by the least 
Table 4. Equivalent widths of molecules found in carbon-rich stars. Date (ddmmyy) shows the observing date of ISAAC or ISO/SWS, which is different from that of photometric observations.

\begin{tabular}{|c|c|c|c|c|c|c|c|}
\hline Name & Date & $\phi_{\mathrm{opt}}$ & $\begin{array}{c}E W_{3.1} \\
\left(\times 10^{-2}\right) \\
{[\AA]}\end{array}$ & $\begin{array}{c}E W_{3.5} \\
{[\AA]} \\
\end{array}$ & $\begin{array}{c}E W_{3.8} \\
\left(\times 10^{-2}\right) \\
{[\AA]}\end{array}$ & {$[\mathrm{mag}]$} & $\begin{array}{c}K \\
{[\mathrm{mag}]} \\
\end{array}$ \\
\hline \multicolumn{8}{|l|}{$L M C$} \\
\hline IRAS 04286-6937 & $14-12-01$ & & $7.00 \pm 0.08$ & $-2.10 \pm 0.87$ & $3.626 \pm 0.003$ & $13.00 \pm 0.05$ & $11.25 \pm 0.05$ \\
\hline IRAS 04496-6958 & $12-12-01$ & & $12.26 \pm 0.02$ & $7.82 \pm 0.63$ & $7.167 \pm 0.005$ & $10.90 \pm 0.05$ & $9.50 \pm 0.04$ \\
\hline IRAS 04539-6821 & $13-12-01$ & & $6.55 \pm 0.03$ & $1.37 \pm 0.48$ & $1.803 \pm 0.002$ & $14.30 \pm 0.05$ & $11.80 \pm 0.04$ \\
\hline IRAS 04557-6753 & $13-12-01$ & & $10.84 \pm 0.03$ & $1.08 \pm 0.42$ & $5.556 \pm 0.002$ & $14.60 \pm 0.05$ & $12.40 \pm 0.04$ \\
\hline IRAS 05112-6755 & $15-12-01$ & & $8.37 \pm 0.04$ & $-0.01 \pm 0.69$ & $2.500 \pm 0.004$ & $14.70 \pm 0.10$ & $12.00 \pm 0.05$ \\
\hline SHV 0521050-69-04-15 & $15-12-01$ & & $13.12 \pm 0.06$ & $12.87 \pm 1.22$ & $1.068 \pm 0.009$ & $9.77 \pm 0.02$ & $9.23 \pm 0.02$ \\
\hline LI-LMC 1813 & $13-12-01$ & & $11.10 \pm 0.18$ & $14.05 \pm 0.22$ & $2.065 \pm 0.005$ & & \\
\hline \multicolumn{8}{|l|}{$S M C$} \\
\hline SMC-S30 & $24-07-02$ & & $14.58 \pm 0.00$ & $-0.79 \pm 0.16$ & $5.944 \pm 0.001$ & $13.48 \pm 0.03$ & $11.42 \pm 0.03^{4}$ \\
\hline \multicolumn{8}{|l|}{ SgrD } \\
\hline Sgr-C3 & $24-07-02$ & & $6.96 \pm 0.01$ & $1.29 \pm 0.28$ & $0.033 \pm 0.002$ & 10.02 & $9.44^{5}$ \\
\hline Sgr-UKST3 & 24-07-02 & & $7.94 \pm 0.02$ & $1.83 \pm 0.36$ & $0.017 \pm 0.002$ & 10.13 & $9.51^{5}$ \\
\hline Sgr-UKST15 & $24-07-02$ & & $2.54 \pm 0.01$ & $0.74 \pm 0.38$ & $1.360 \pm 0.002$ & 10.08 & $9.65^{5}$ \\
\hline \multicolumn{8}{|l|}{ Galactic carbon-rich stars } \\
\hline LP And & $02-12-96$ & & 3.44 & 3.85 & -0.46 & $6.355 \pm 0.018^{7}$ & $3.859 \pm 0.036^{7, *}$ \\
\hline VX And & $17-01-97$ & & 12.42 & -0.21 & 0.07 & $1.891 \pm 0.202$ & $1.193 \pm 0.202^{7}$ \\
\hline V Aql & $29-04-96$ & & 8.71 & 1.10 & 0.48 & 0.64 & -0.09 \\
\hline WZ Cas & $22-07-96$ & & 10.84 & 9.85 & -0.37 & 1.01 & $0.57^{3}$ \\
\hline S Cep & $31-05-97$ & & 6.44 & 3.60 & 2.07 & $1.013 \pm 0.186$ & $-0.029 \pm 0.192^{7}$ \\
\hline \multirow[t]{6}{*}{ V CrB } & 07-03-96 & 0.20 & 8.75 & 10.37 & 3.08 & $2.209 \pm 0.218$ & $1.321 \pm 0.276^{7}$ \\
\hline & $29-07-96$ & 0.17 & 8.56 & 9.90 & 3.07 & & \\
\hline & $11-01-97$ & 0.64 & 7.77 & 6.28 & 2.00 & & \\
\hline & 06-03-97 & 0.19 & 5.16 & 2.01 & 0.35 & & \\
\hline & $12-06-97$ & 0.78 & 7.32 & 4.12 & 1.45 & & \\
\hline & 21-09-97 & 0.32 & 8.88 & 9.22 & 2.18 & & \\
\hline Y CVn & $25-04-96$ & & 8.77 & & 1.13 & $-0.285 \pm 0.166$ & $-0.738 \pm 0.166^{7}$ \\
\hline V Cyg & $05-02-96$ & 0.52 & 9.26 & 10.05 & 3.44 & $1.273 \pm 0.198$ & $0.117 \pm 0.192^{7}$ \\
\hline \multirow[t]{2}{*}{ V460 Cyg } & $11-01-97$ & & 4.66 & & 1.14 & $0.583 \pm 0.192$ & $0.270 \pm 0.184^{7}$ \\
\hline & $29-11-97$ & & 3.02 & & 1.00 & & \\
\hline RY Dra & $12-05-97$ & & 7.74 & 1.38 & 0.72 & $0.955 \pm 0.214$ & $0.394 \pm 0.204^{7}$ \\
\hline \multirow[t]{8}{*}{ T Dra } & $07-03-96$ & 0.59 & 7.29 & 12.86 & 4.33 & $2.454 \pm 0.192$ & $1.365 \pm 0.212^{7}$ \\
\hline & $21-07-96$ & 0.93 & 7.81 & 8.21 & 3.09 & & \\
\hline & $28-10-96$ & 0.17 & 6.98 & 6.78 & 2.27 & & \\
\hline & $04-12-96$ & 0.25 & 7.51 & 11.69 & 3.74 & & \\
\hline & $18-01-97$ & 0.36 & 5.67 & 4.68 & 1.64 & & \\
\hline & $26-01-97$ & 0.38 & 6.15 & 4.46 & 1.81 & & \\
\hline & $15-05-97$ & 0.64 & 7.77 & 7.88 & 2.89 & & \\
\hline & $21-08-97$ & 0.86 & 5.65 & 8.87 & 2.80 & & \\
\hline R For & $13-02-98$ & & 6.83 & 0.91 & 1.85 & $2.537 \pm 0.206$ & $1.349 \pm 0.274^{7}$ \\
\hline CW Leo & $31-05-96$ & & 1.81 & 1.05 & -0.33 & 4.25 & $1.28^{2}$ \\
\hline W Ori & $22-03-98$ & & 4.90 & 0.01 & 0.26 & $0.092 \pm 0.338$ & $-0.470 \pm 0.402^{7}$ \\
\hline RZ Peg & $10-06-96$ & & 9.08 & 13.15 & 1.98 & $2.850 \pm 0.214$ & $2.127 \pm 0.302^{7}$ \\
\hline TX Psc & $26-11-96$ & & 5.34 & 2.35 & 0.87 & -0.33 & $-0.67^{3}$ \\
\hline $\mathrm{R} \mathrm{Scl}$ & $28-11-96$ & 0.28 & 13.47 & 6.92 & 2.09 & 0.75 & $-0.07^{2}$ \\
\hline S Sct & $29-04-96$ & & 9.06 & 4.18 & 2.06 & 1.06 & $0.44^{3}$ \\
\hline SZ Sgr & $28-03-98$ & & 3.72 & & 1.19 & $2.623 \pm 0.216$ & $2.168 \pm 0.234^{7}$ \\
\hline
\end{tabular}


Table 4. continued.

\begin{tabular}{|c|c|c|c|c|c|c|c|}
\hline Name & Date & $\phi_{\mathrm{opt}}$ & $\begin{array}{c}E W_{3.1} \\
\left(\times 10^{-2}\right) \\
{[\AA]}\end{array}$ & $\begin{array}{c}E W_{3.5} \\
{[\AA]}\end{array}$ & $\begin{array}{c}E W_{3.8} \\
\left(\times 10^{-2}\right) \\
{[\AA]}\end{array}$ & [mag] & [mag] \\
\hline TU Tau & $18-03-98$ & & 4.78 & 1.67 & 1.16 & $2.093 \pm 0.210$ & $1.574 \pm 0.220^{7}$ \\
\hline RU Vir & $20-07-96$ & & 4.86 & 6.88 & 1.50 & 2.50 & $1.33^{3}$ \\
\hline SS Vir & $14-06-96$ & & 14.80 & 6.80 & 3.80 & $1.811 \pm 0.234$ & $0.831 \pm 0.330^{7}$ \\
\hline AFGL 940 & 04-04-98 & & 1.75 & 2.32 & -0.16 & $6.269 \pm 0.013$ & $4.391 \pm 0.011^{7}$ \\
\hline AFGL 2155 & 01-03-97 & & 3.38 & 3.05 & 0.51 & $8.722 \pm 0.038$ & $5.609 \pm 0.018^{7}$ \\
\hline AFGL 2392 & $22-03-98$ & & 4.87 & 7.32 & 0.34 & 5.24 & $3.65^{2}$ \\
\hline CS 2178 & $25-01-97$ & & 7.52 & & 2.05 & $4.158 \pm 0.172$ & $2.798 \pm 0.234^{7}$ \\
\hline CS 2429 & $20-02-97$ & & 6.18 & & 0.19 & $1.699 \pm 0.342$ & $1.007 \pm 0.346^{7}$ \\
\hline DO 40123 & $15-01-97$ & & 11.74 & 8.69 & 3.35 & $1.867 \pm 0.182$ & $0.913 \pm 0.192^{7}$ \\
\hline IRC-10095 & 01-04-98 & & 13.94 & 1.23 & 2.84 & $3.235 \pm 0.206$ & $2.241 \pm 0.248^{7}$ \\
\hline IRC-10122 & $31-03-98$ & & 6.01 & 2.07 & 1.25 & $3.851 \pm 0.248$ & $2.399 \pm 0.272^{7}$ \\
\hline IRC +00365 & $29-03-97$ & & 7.17 & 4.49 & 1.69 & $4.100 \pm 0.182$ & $2.209 \pm 0.242^{7}$ \\
\hline IRC +50096 & $02-02-98$ & & 5.22 & 5.15 & 1.69 & $2.596 \pm 0.192$ & $1.150 \pm 0.192^{7}$ \\
\hline IRAS $19068+0544$ & 09-03-97 & & 6.06 & & 2.51 & $4.685 \pm 0.075$ & $3.080 \pm 0.254^{7}$ \\
\hline
\end{tabular}

1: Gezari et al. (1993); 2: Le Bertre (1992) (average over the all phase); 3: Noguchi et al. (1981); 4: Groenewegen \& Blommaert (1998); 5: Whitelock et al. (1996); 6: Trams et al. (1999b); 7: 2MASS; *: 2MASS quality flag is "E", i.e., poor quality.

abundant component, Si. Silicon is an $\alpha$-element, not synthesized in AGB stars; the Si abundance reflects the one at the time the progenitor star was formed. In supergiants, $\mathrm{Si}$ is formed but only in the last stage of their evolution. The current Si abundance in the LMC can be measured from H II regions or from hot stars. The solar abundance is $\log (\mathrm{Si} / \mathrm{H})+12=7.6$ (Anders \& Grevesse 1989). LMC H II regions show $\log (\mathrm{Si} / \mathrm{H})+12=7.7$ (Russell \& Dopita 1992), and main sequence B-type stars have 6.7 (Garnett 1999). For planetary nebulae Dopita et al. (1997) found $\log (\mathrm{Si} / \mathrm{H})+12=6.5-7.7$ : the large difference may reflect the range in progenitor ages. Here, we use $\log (\mathrm{Si} / \mathrm{H})+12=7.0$ for the LMC late-type stars, taken from an average over the planetary nebulae (PNe).

Aringer et al. (1997) calculated the $E W$ of $\mathrm{SiO}$ bands in M-type giants using a hydrostatic model. $E W_{\mathrm{SiO}}$ is sensitive to both the effective temperature and silicon abundance. Aringer et al. (1999) measured the $\mathrm{SiO} E W$ of Galactic stars, finding values ranging from $0-50 \AA$. Extrapolating this linearly to the LMC metallicity yields $E W_{\mathrm{SiO}}$ of $0-30 \AA$ (Fig. 12). The low $\mathrm{Si}$ elemental abundance could be the major cause of the lower $E W_{\mathrm{SiO}}$ observed in the LMC.

Our measured $E W_{\mathrm{SiO}}$ limits for three stars are at the lower range of the extrapolation from Aringer et al. (1997, 1999). Galactic stars show a spread in $E W_{\mathrm{SiO}}$; for the LMC stars we can also expect a few very low values. The low upper limits can be interpreted as evidence that the $\mathrm{Si}$ abundance among LMC oxygen-rich stars is lower than the assumed value of 7.0. However, two other possible reasons should be considered.

First, for Galactic stars Aringer et al. (1999) reported that observed $E W_{\mathrm{SiO}}$ are lower than their model predictions, with Mira variables showing weaker bands than semi-regular variables. Tsuji et al. (1994), who observed M-giants and supergiants, suggest that the strong pulsations extend the atmosphere

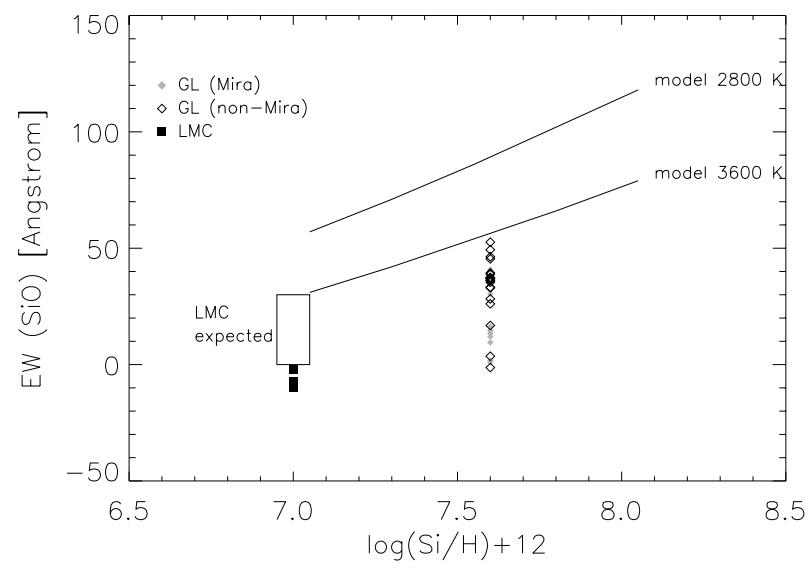

Fig. 12. The equivalent width of $\mathrm{SiO}$ bands as a function of metallicity. The model is from Aringer et al. (1997), and Galactic data are from Aringer et al. (1999).

above the photosphere. Molecular bands in this extended atmosphere may fill in their photospheric counterparts. In extreme cases, the $\mathrm{SiO}$ bands could be in emission during the maximum phase of the variability (Yamamura et al. 1999), when the radius of the extended atmosphere is largest (Matsuura et al. 2002a). At least two of the LMC targets are Mira variables (Whitelock et al. 2003). We may have observed the stars during the phase of the pulsation cycle when the $\mathrm{SiO}$ bands are weak. For two stars, for instance, we know the approximate phase at the time of observation: they were observed near maximum phase, when $\mathrm{SiO}$ bands could be weak.

The second possible explanation is that dust emission suppresses the $\mathrm{SiO}$ equivalent width. Among our Galactic sample, red stars (VY Sgr and VY CMa) with $H-K>0.7$ mag do not show any trace of $\mathrm{SiO}$ bands. In our LMC sample, two stars 
have comparably high values in $H-K$ and could be affected by dust emission. Therefore, we suggest that the dust filling could decrease the $E W_{\mathrm{SiO}}$.

We also investigated the effect of higher $\mathrm{C} / \mathrm{O}$ abundance for LMC stars, as dredge-up of newly produced carbon (3rd dredge-up) has a larger effect for low metallicity stars (see next section) and would reduce the free oxygen after the initial formation of CO. However, less oxygen has little or no effect on the $\mathrm{SiO}$ abundance because the $\mathrm{Si}$ abundance still limits the $\mathrm{SiO}$ formation. Only for $\mathrm{C} / \mathrm{O}$ very near to unity (i.e. for $\mathrm{S}$ or SC stars) some effect may be expected (Zijlstra et al. 2004).

In conclusion, within our limited sample, we did not detect $\mathrm{SiO}$ bands at all. The model predicts that low $\mathrm{Si}$ abundance results in the low $\mathrm{SiO}$ abundance, and our observations do not conflict with this trend. The measured $E W_{\mathrm{SiO}}$ in the LMC is even lower than the expected range of $E W_{\mathrm{SiO}}$. Although several explanations, such as pulsations and dust filling, can be given for the lower $E W_{\mathrm{SiO}}$ than expected in the LMC, the issue is not settled. The solution awaits observation of a larger number of stars with complete coverage in colour both in our Galaxy and in the LMC.

\section{2. $\mathrm{C}_{2} \mathrm{H}_{2}, \mathrm{CH}$ and carbon abundances of carbon-rich stars}

The $3.8 \mu \mathrm{m} \mathrm{C}_{2} \mathrm{H}_{2}$ band is found in only a few ISO/SWS spectra of Galactic carbon-rich stars: V Cyg and HD 192443 (Matsuura et al. 2002b; Vandenbussche et al. 2002). On the other hand, almost all red LMC or SMC carbon stars show this band in their spectra. The measured $E W_{3.8}$ is clearly larger among red carbon stars in the LMC, compared to the galactic stars. One SMC star measured in this work also follows this trend.

The likely explanation for the stronger $3.8 \mu \mathrm{m}$ band is that the LMC and SMC stars have a higher $\mathrm{C} / \mathrm{O}$ ratio (Paper I). If all the elemental abundances simply scale with the metallicity, the $\mathrm{C}_{2} \mathrm{H}_{2}$ abundance should be less because fewer carbon atoms are available. The $\mathrm{C} / \mathrm{O}$ ratio of the star, initially less than unity, increases during the third dredge-up on the thermal-pulsing AGB. A fixed amount of carbon injected into the envelope has a larger effect for a low metallicity, leading to a higher C/O ratio (Lattanzio \& Wood 2003; Mouhcine \& Lançon 2003). Additionally, the third dredge-up operates more efficiently at lower metallicity (Vassiliadis \& Wood 1993), adding larger quantities of carbon. Both effects predict a systematically higher $\mathrm{C} / \mathrm{O}$ ratio in the $\mathrm{LMC}$ and the SMC. In stars with $\mathrm{C} / \mathrm{O}>1$ almost all the oxygen is tied up in $\mathrm{CO}$, and the excess carbon will be available for forming carbon-bearing molecules.

A higher $\mathrm{C} / \mathrm{O}$ ratio at low metallicity is supported by the fact that the ratio of numbers of carbon-rich stars over oxygen-rich stars $\left(N_{\mathrm{C}} / N_{\mathrm{O}}\right)$ increases with decreasing metallicity of the host galaxy (Groenewegen 1999). Further confirmation comes from LMC and SMC planetary nebulae, which show a higher C/O ratio compared to Galactic PNe (Leisy \& Dennefeld 1996). PNe show the final AGB abundances after the last dredge-up event.
Cohen et al. (1981) found that the $J-K$ colour is redder in LMC carbon stars than in Galactic carbon stars. They suggested that the $J$-band magnitude in LMC stars is suppressed by deeper $\mathrm{C}_{2}$ absorption, and also mention a higher $\mathrm{C} / \mathrm{O}$ as the likely cause. The stronger $\mathrm{C}_{2} \mathrm{H}_{2}$ absorption is consistent with this.

A chemical equilibrium model (Fig. 13) shows increase of the $\mathrm{C}_{2} \mathrm{H}_{2}$ fractional abundance as function of the $\mathrm{C} / \mathrm{O}$ ratio. Details of the model are given in Markwick (2000). Chemical equilibrium is a reasonable assumption for the stellar photosphere but could be questioned for the lower density atmosphere. We assume a typical $\mathrm{C} / \mathrm{O}$ ratio for Galactic carbon stars of 1.2 (Lambert et al. 1986; Ohnaka et al. 2000). To obtain a higher $\mathrm{C}_{2} \mathrm{H}_{2}$ fractional abundance for the LMC stars requires $\mathrm{C} / \mathrm{O} \sim 1.4$, while the SMC carbon-rich stars should have an even higher value.

The mean $\mathrm{C} / \mathrm{O}$ ratio found in carbon-rich $\mathrm{PNe}$ in the $\mathrm{LMC}$ is 3.2 (Leisy \& Dennefeld 1996,8 samples) or 2.8 (Dopita et al. 1997, 4 samples). AGB stars may experience several more third dredge-up events, giving a higher $\mathrm{C} / \mathrm{O}$ ratio in $\mathrm{PNe}$ than in AGB stars. However, we cannot exclude such a high $\mathrm{C} / \mathrm{O}$ ratio in the LMC AGB stars. The $\mathrm{C}_{2} \mathrm{H}_{2}$ abundance increases only slowly for $\mathrm{C} / \mathrm{O}>1.4$ (Fig. 13); the relative increase in the number of free carbon atoms (after $\mathrm{CO}$ formation) is fastest for $\mathrm{C} / \mathrm{O}$ close to unity.

In Fig. 10, the $E W$ of $\mathrm{C}_{2} \mathrm{H}_{2}$ increases sharply at $H-K \approx 0.5$, and declines above 1.2. The initial increase is caused by a temperature effect. $\mathrm{C}_{2} \mathrm{H}_{2}$ is favoured at lower temperatures: at higher temperatures more of the carbon is in diatomic molecules. The temperature dependence of the various species is shown in Fig. 14. The decay at redder $H-K$ colour is explained by additional circumstellar emission.

We also detect $\mathrm{CH}$ bands in the infrared spectra, for carbonrich stars in the LMC, the SMC, and SgrD. The CH abundance depends on the C/O ratio: Aoki et al. (1998) found that SC-type stars, which have $\mathrm{C} / \mathrm{O}$ ratio close to one, show only weak $\mathrm{CH}$ lines, while $\mathrm{N}$-type stars show clear $\mathrm{CH}$ bands. Figure 13 shows that $\mathrm{CH}$ increases more slowly with $\mathrm{C} / \mathrm{O}$ ratio than does $\mathrm{C}_{2} \mathrm{H}_{2}$, as expected for its single carbon atom. For a Galactic ratio of $\mathrm{C} / \mathrm{O}=1.2$, the $\mathrm{CH}$ abundances in the $\mathrm{LMC}$ and the Galaxy may become comparable but one would not expect much stronger bands in the LMC. The same argument holds for the SMC. We have not made a comparison in line strengths of the $\mathrm{CH}$ bands because they are blended with other molecular bands. However, the fact that these bands are seen in all stars including the Galactic ones qualitatively supports the suggestion that the $\mathrm{CH}$ band strength is relatively independent of metallicity.

In conclusion, a high $\mathrm{C} / \mathrm{O}$ ratio affects the spectra of extragalactic carbon-rich stars. The $\mathrm{C} / \mathrm{O}$ ratio should be treated as an important parameter for spectral classification.

\subsection{HCN and nitrogen abundance of carbon-rich stars}

In contrast to the $\mathrm{C}_{2} \mathrm{H}_{2}$ and possibly $\mathrm{CH}$, the $\mathrm{HCN}$ lines are generally very weak in the extra-galactic stars. Lloyd Evans (1980) finds that optical carbon stars in the LMC show weak 

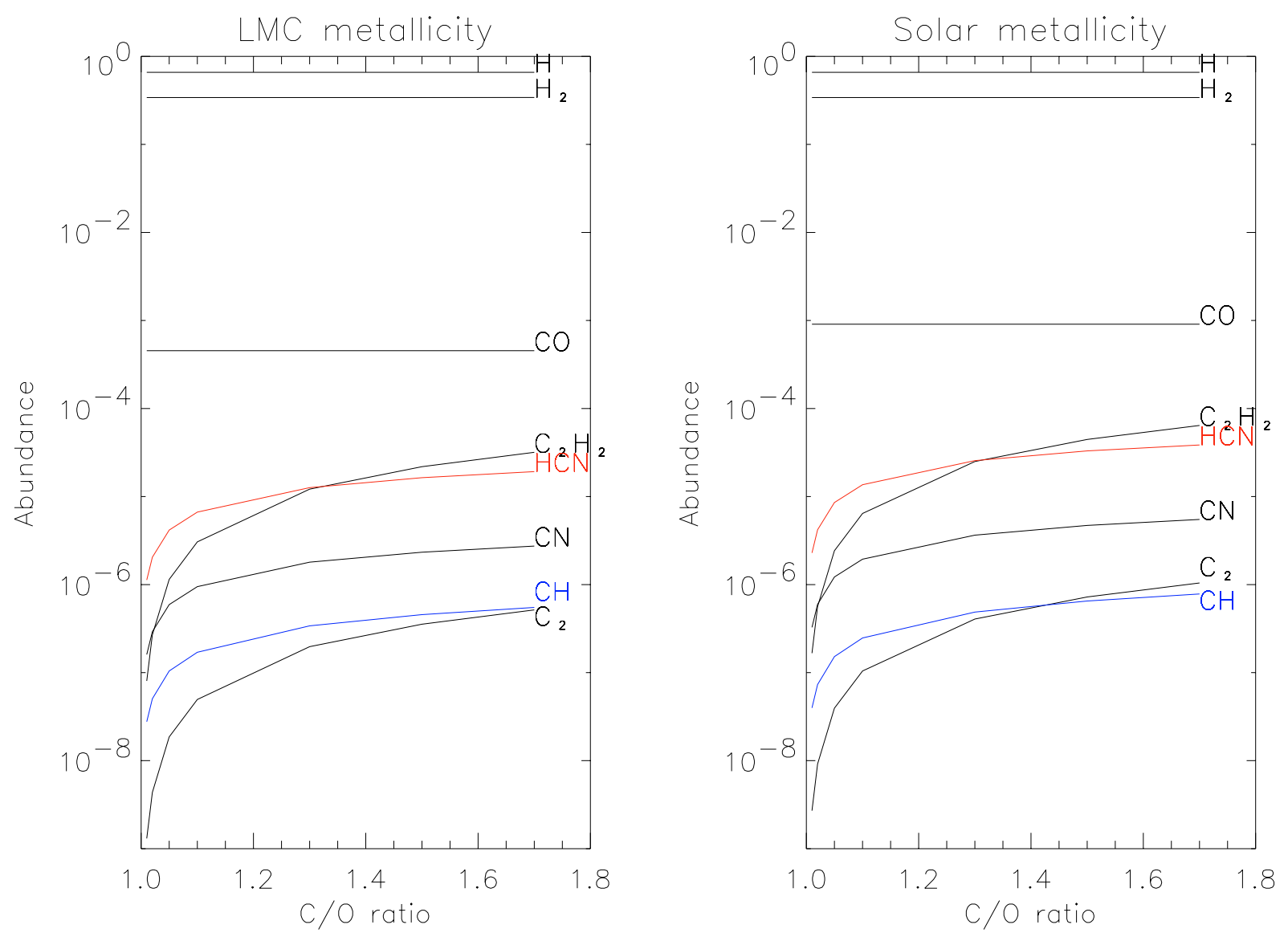

Fig. 13. Abundance of molecules calculated with the chemical model. The abundance is normalized by $\mathrm{H}+\mathrm{H}_{2}$ pressure. The left side is for $[\mathrm{Z} / \mathrm{H}]=-0.3$ (approximate LMC metallicity), and the right for $[\mathrm{Z} / \mathrm{H}]=0.0$ (solar metallicity). All elemental abundances are scaled to the metallicity, except carbon, which is set relative to the oxygen abundance using the $\mathrm{C} / \mathrm{O}$ ratio. The temperature is $2500 \mathrm{~K}$ for both panels.

(optical) $\mathrm{CN}$ bands compared to $\mathrm{C}_{2}$. This argues against temperature as a factor, since $\mathrm{C}_{2}$ and $\mathrm{CN}$ show very similar temperature dependence (Fig. 14), as do (to a lesser extent) $\mathrm{C}_{2} \mathrm{H}_{2}$ and $\mathrm{HCN}$. This leaves the nitrogen abundance as the most likely explanation.

Although the majority of extra-galactic carbon stars do not show the $3.5 \mu \mathrm{m}$ HCN feature, a few LMC stars have $E W_{3.5}>$ $5 \AA$ as high as Galactic stars. There may be two discrete extragalactic groups: $\mathrm{HCN}$-rich and non-HCN. The $\mathrm{C} / \mathrm{N}$ ratio should be different for these two groups.

The $\mathrm{C} / \mathrm{N}$ ratio varies strongly over the course of the star's evolution, mainly because of $\mathrm{C}$ production and $\mathrm{CN}$ cycling which transmutes $\mathrm{C}$ into N. First dredge-up decreases carbon and increases nitrogen, and lowers the $\mathrm{C} / \mathrm{N}$ ratio by a factor of 2 or more. The largest changes affect stars more massive than 2-3 $M_{\odot}$. Second dredge-up, which only occurs in stars with $M \gtrsim 4 M_{\odot}$, has a similar effect, and alters the $\mathrm{C} / \mathrm{N}$ ratio by a factor of $\sim 1.5$. Third dredge-up increases the carbon abundance by large factors but leaves $\mathrm{N}$ relatively unaffected. The most massive AGB stars may suffer burning at the bottom of the convective envelope, a process called hot bottom burning which again cycles $C$ into $N$. The cumulative changes in the $\mathrm{C} / \mathrm{N}$ ratios are shown in Boothroyd \& Sackman (1999).
The effect of the third dredge-up scenario can explain two distinct groups: low-metallicity stars without hot bottom burning (HBB; Iben 1981) will show low N, while stars with HBB show enhanced N. An example of an LMC star believed to have experienced HBB is IRAS 04496-6958, which is a high-luminosity silicate carbon star (Trams et al. 1999a).

The fact that 3 of our 8 LMC stars are $\mathrm{HCN}$-rich suggests that the lower mass limit for HBB cannot be much higher than the canonical $4 M_{\odot}$, since otherwise very few AGB stars would pass through this phase. In PNe, nitrogen and carbon elemental abundance ratios vary greatly between individual objects (Dopita et al. 1997), showing the effect of the initial parameters on the enrichment history.

\subsection{Grain formation and mass-loss at lower metallicity}

Our observations suggest that at low metallicities, carbon and oxygen-rich stars behave differently. Carbon stars show higher $\mathrm{C} / \mathrm{O}$ ratios and more abundant carbon-bearing molecules (excluding $\mathrm{CO}$ ) than their Galactic counterparts. Oxygen-rich stars show primarily low $\mathrm{SiO}$ abundances, as expected from the low metallicity. This difference can be expected to affect their dust formation, since $\mathrm{C}_{2} \mathrm{H}_{2}$ and $\mathrm{SiO}$ are the respective starting 

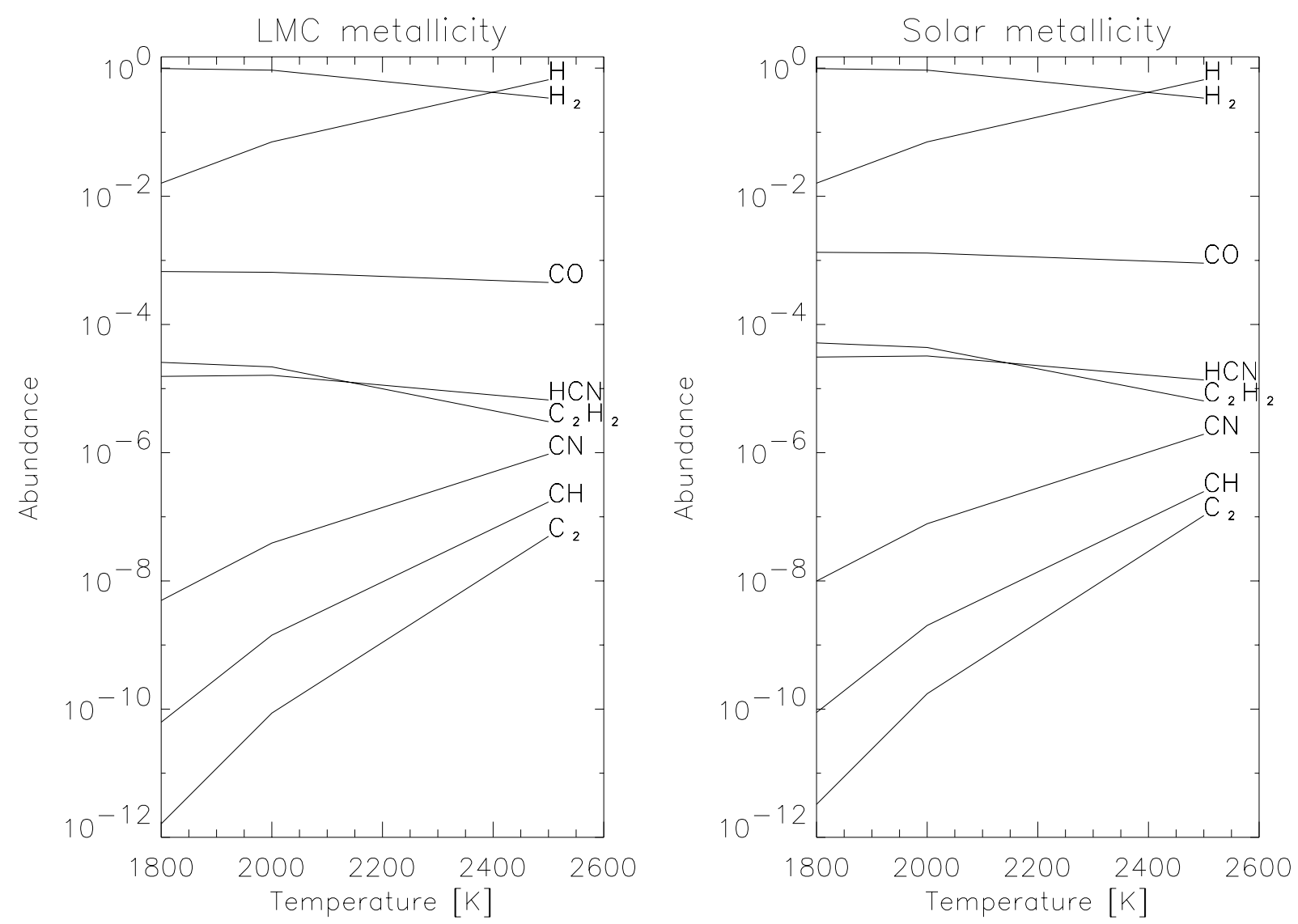

Fig. 14. Same as Fig.13, but as a function of temperature. The $\mathrm{C} / \mathrm{O}$ ratio is 1.1 for both panels.

points for build-up towards either carbonaceous or silicate dust grains.

For oxygen-rich stars, silicate grains require $\mathrm{Si}, \mathrm{O}$ and iron and/or magnesium. The low $\mathrm{SiO}$ abundance caused by the low metallicity suggests a lower silicate dust abundance. Iron or magnesium may also limit the amount of silicate dust.

For carbon stars, Groenewegen (1995) shows that the mass of amorphous carbon dust is about 10 times more than that of silicon carbide (SiC). Large molecules can build up from $\mathrm{C}_{2} \mathrm{H}_{2}$ (Woods et al. 2002) and can form a major component of carbonaceous dust, as shown by the large mass of carbon dust. The enhanced $\mathrm{C} / \mathrm{O}$ ratio, giving a high abundance of $\mathrm{C}_{2} \mathrm{H}_{2}$, can allow for carbonaceous dust to form at a rate at least comparable to those of Galactic stars. In addition, if PAHs may be formed in AGB stars (but not excited), then more $\mathrm{C}_{2} \mathrm{H}_{2}$ can also lead to enhanced PAH abundances.

The dust formation rate directly affects the mass-loss rate of the star. Above a metallicity of $[\mathrm{Fe} / \mathrm{H}]=-1.0$, the massloss process is driven via radiation pressure on the dust - the accelerated dust drags the gas with it (Bowen \& Willson 1991). At low metallicities the amount of dust formed will be very small and mass loss will be very inefficient.

This all suggests that while oxygen-rich stars will show low mass-loss rates due to the reduced $\mathrm{SiO}$, carbon stars should be able to reach mass-loss rates similar to Galactic stars, which is consistent with our (limited) knowledge of LMC stars.
Carbon stars in the LMC reach mass-loss rates of a few times $10^{-5} M_{\odot} \mathrm{yr}^{-1}$ (van Loon 2000), similar to what is found in the Galaxy. Oxygen-rich stars in the LMC reach higher massloss rates than Galactic stars (van Loon 2000), but do so at a luminosity several times higher than the LMC carbon stars. Direct comparison with Galactic stars is hampered by uncertain distances to Galactic stars and by the fact that the LMC measurements were made for stars known to be surrounded by dust, so that the sample is already biased towards high mass-loss rates.

If oxygen-rich stars show reduced mass-loss but carbon stars do not, this will affect the composition of the dust which subsequently enters the ISM. Even if oxygen-rich stars can still reach the mass-loss rates, but do so later in their evolution, this would still imply a shift in composition since the delay will allow more stars to reach the carbon star phase. The main sources of dust in a galaxy are supernovae (and their progenitors) and AGB stars, but the relative contribution of each is uncertain and will differ for silicate and carbonaceous dust. Reduced mass-loss of oxygen-rich AGB stars will cause a relative shift towards carbonaceous grains. The ISO observations of $\mathrm{HII}$ regions and molecular clouds show that PAH bands are ubiquitous in the LMC and the SMC (Reach et al. 2000; Vermeij et al. 2002), with a similar fraction to total dust as found in our Galaxy; the detailed composition of the PAHs may vary with more open PAHs found in the SMC. The fractional 
dust abundance in the SMC is 30 times lower than that of our Galaxy, but although this holds for both small and large grains (Bot et al. 2004), it is not known whether the fractions of carbonaceous and silicate dust differ. Our detection of a PAH band from an SMC post-AGB star shows that PAHs can form in a low-metallicity AGB wind.

\section{Conclusion}

We report the strength of infrared molecular bands in AGB stars in the LMC, SMC and SgrD galaxies. The metallicities range from -0.3 to -0.7 . Carbon stars are observed in all three galaxies. The LMC and the SMC contain luminous and high mass-loss stars that are likely to be young, intermediatemass AGB stars. The SgrD stars are older with a likely age around 5 Gyr and are optical carbon stars without evidence of high mass loss.

Our main conclusions are:

- The oxygen-rich stars in the sample show weak or no $\mathrm{SiO}$ bands. We have good spectra for 3 stars at medium resolution, a lower quality spectrum for one star, and two further spectra at low resolution, all in the LMC. At low metallicity, weaker $\mathrm{SiO}$ bands are expected, and our measurements do not conflict with the metallicity effects on $\mathrm{SiO}$ bands. However, in our limited sample, factors such as dust or pulsation could hamper the correct measurements of $\mathrm{SiO}$ bands in the LMC.

- The carbon-rich stars in the LMC and the SMC show very strong $\mathrm{C}_{2} \mathrm{H}_{2}$ bands, when compared to Galactic stars, which implies a very high abundance of this molecule is implied. The likely explanation is a high $\mathrm{C} / \mathrm{O}$ ratio of low-metallicity carbon stars, leading to a larger amount of free carbon. The high $\mathrm{C} / \mathrm{O}$ ratio is supported by data for planetary nebulae. Chemical equilibrium abundances are presented for a range of $\mathrm{C} / \mathrm{O}$ ratios, showing that high $\mathrm{C}_{2} \mathrm{H}_{2}$ can be reproduced with $\mathrm{C} / \mathrm{O} \gtrsim 1.4$.

- The HCN band is very weak compared to Galactic stars, except for a few LMC stars that show strong HCN. This could imply a difference in initial mass. We show that a low abundance of N-bearing molecules is expected for lowmetallicity stars that do not experience hot bottom burning. The two populations are likely to be separated in mass, since only stars with progenitor masses $\gtrsim 4 M_{\odot}$ are likely to experience hot bottom burning.

- CH bands are seen in stars from all galaxies, including our own. The chemical equilibrium calculations show that this diatomic molecule is less affected by the $\mathrm{C} / \mathrm{O}$ ratio, so that similar abundances in all the observed systems can be expected. The lines are blended with other molecular bands, and agreement with the models is only qualitative.

- We detect a PAH $3.3 \mu \mathrm{m}$ band in a post-AGB star in the SMC. This may be the first detection of PAHs in such a star. PAH formation proceeds even in low-metallicity evolved stars.

- The difference in abundance behaviour between carbon and oxygen stars is expected to affect their dust formation and mass-loss rates. In oxygen-rich stars, silicate dust forms, which should be directly affected by the possible low abundance of SiO. Carbon stars form carbonaceous and hydrocarbon dust from molecules that we find to have a high abundance. The mass-loss from AGB stars is driven by radiation pressure on the dust, which leads us to expect that low-metallicity carbon stars will show mass-loss rates as high as Galactic stars, but oxygen-rich stars will tend to show lower mass-loss rates at the same luminosity. The limited available data indicates that some oxygen-rich stars exist in the LMC with similar mass-loss rates to carbon stars, but they are several times more luminous than the carbon stars. A comprehensive survey of mass-loss rates from LMC AGB stars is not yet available, but it could confirm whether less luminous oxygen-rich stars in the LMC indeed show low mass-loss rates.

- We predict that dust input from AGB stars into the ISM contains a higher fraction of carbonaceous grains than is the case for our Galaxy. Whether this affects the ISM dust depends on the relative contribution from AGB stars compared to other dust sources, primarily supernovae. $\mathrm{PAH}$ emission is ubiquitous in the SMC, and our detection in the post-AGB star shows that evolved stars are a potential source of PAHs.

Acknowledgements. We would like to thanks Dr. Y. Jung for helping with our data reduction. The ISO data archive and the 2MASS data archive provide useful data for this study. Simbad data base is used for this research. M.M. is supported by a PPARC Rolling grant.

\section{References}

Anders, E., \& Grevesse, N. 1989, Geochim. Cosmochim. Acta, 53, 197

Aoki, W., Tsuji, T., \& Ohnaka, K. 1998, A\&A, 340, 222

Aringer, B., Jørgensen, U. G., \& Langhoff, S. R. 1997, A\&A, 323, 202

Aringer, B., Höfner, S., Wiedemann, G., et al. 1999, A\&A, 342, 799

Boothroyd, A. I., \& Sackman, I.-J. 1999, ApJ, 510, 232

Bot, C., Boulanger, F., Lagache, G., Cambrésy, L., \& Egret, D. 2004, A\&A, 423, 567

Bowen, G. H., \& Willson, L. A. 1991, ApJ, 375, L53

Cioni, M.-R., Loup, C., Habing, H. J., et al. 2000, A\&AS, 144, 235

Cohen, J. G., Persson, S. E., Elias, J. H., \& Frogel, J. A. 1981, ApJ, 249,481

Dopita, M. A., Vassiliadis, E., \& Wood, P. R., et al. 1997, ApJ, 474, 188

Dudziak, G., Péquignot, D., Zijlstra, A. A., \& Walsh, J. R. 2000, A\&A, 363,717

Edmunds, M. G. 2001, MNRAS, 328, 223

Elias, J. H., Frogel, J. A., \& Schwering, P. B. W. 1986, ApJ, 302, 675

Garnett, D. R. 1999, in New Views of the Magellanic Clouds, ed. Y.-H. Chu, N. Suntzeff, J. Hesser, \& D. Bohlender, IAU Symp., 190, 266

Gezari, D. Y., Schmitz, M. M., \& Jaylee, M. 1993, Catalog of infrared observations, NASA

Goebel, J. H., Bregman, J. D., Witteborn, F. C., Taylor, B. J., \& Willner, S. P. 1981, ApJ, 246, 455

Gordon, K. D., Clayton, G. C., Misselt, K. A., Landolt, A. U., \& Wolff, M. J. 2003, ApJ, 594, 279

Groenewegen, M. A. T. 1995, A\&A, 293, 463

Groenewegen, M. A. T. 1999, Asymptotic Giant Branch Stars, ed. T. Le Bertre, A. Lebre, \& C. Waelkens, IAU Symp., 191, 535 
Groenewegen, M. A. T., \& Blommaert, J. A. D. L. 1998, A\&A, 332, 25

Harris, G. J., Polyansky, O. L., \& Tennyson, J. 2002, ApJ, 578, 657

Harris, G. J., Pavlenko, Ya. V., Jones, H. R. A., \& Tennyson, J. 2003, MNRAS, 344, 1107

Heras, A. M., Shipman, R. F., Price, S. D., et al. 2002, A\&A, 394, 539

Hron, J., Loidl, R., Höfner, S., et al. 1998, A\&A, 335, L69

Hughes, S. M. G. 1989, AJ, 97, 1634

Hughes, S. M. G., \& Wood, P. R. 1990, AJ, 99, 784

Ibata, R. A., Gilmore, G., \& Irwin, M. J. 1995, MNRAS, 277, 781

Iben, I. Jr. 1981, ApJ, 246, 278

Jørgensen, U. G., Larsson, M., Iwamae, A., \& Yu, B. 1996, A\&A, 315, 204

Käufl, H. U., \& Stanghellini, L. 1992, ESO Workshop on High Resolution Spectroscopy with the VLT, ed. M.-H. Ulrich, 135

Lambert, D. L., Gustafsson, B., Eriksson, K., \& Hinkle, K. H. 1986, ApJS, 62, 373

Langhoff, S. R., \& Bauschlicher Jr., C. W. 1993, Chem. Phys. Lett., 211,305

Lattanzio, J. C., \& Wood, P. R. 2003, Asymptotic giant branch stars, ed. H. J. Habing, \& H. Olofsson, Astronomy and astrophysics library, Springer

Leisy, P., \& Dennefeld, M. 1996, A\&AS, 116, 95

Le Bertre, T. 1992, A\&AS, 94, 377

Lloyd Evans, T. 1980, MNRAS, 193, 97

Marconi, G., Buonanno, R., Castellani, et al. 1998, A\&A, 330, 453

Marigo, P. 2002, A\&A, 387, 507

Markwick, A. J. 2000, Ph.D. Thesis, UMIST

Marshall, J. R., van Loon, J. Th., Matsuura, M., et al. MNRAS, in press

Matsuura, M., Yamamura, I., Cami, J., Onaka, T., \& Murakami, H. 2002a, A\&A, 383, 972

Matsuura, M., Zijlstra, A. A., van Loon, J. Th., et al. 2002b, ApJ, 580, L133 (Paper I)

Mouhcine, M., \& Lançon, A. 2003, MNRAS, 338, 572

Noguchi, K., Kawara, K., Kobayashi, Y., et al. PASJ, 33, 373

Ohnaka, K., Tsuji, T., \& Aoki, W. 2000, A\&A, 353, 528

Payne-Gaposchkin, C. H. 1971, The variable stars of the Large Magellanic Cloud Smithsonian Contributions to Astrophysics, Washington

Reach, W. T., Boulanger, F., Contursi, A., \& Lequeux, J. 2000, A\&A, 361, 895

Reid, N., Tinney, C., \& Mould, J. 1990, ApJ, 348, 98

Ridgway, S. T., Carbon, D. F., Hall, D. N. B., \& Jewell, J. 1984, ApJS, 54, 177

Rothman, L. S., Wattson, R. B., Gamache, R. R., et al. 1998, J. Quant. Spec. Radiat. Transf., 60, 665

Russell, S. C., \& Dopita, M. A. 1992, ApJ, 384, 508

Sauval, A. J., \& Tatum, J. B. 1984, ApJS, 56, 193
Schwering, P. B. W., \& Israel, F. P. 1990, Atlas and catalogue of infrared sources in the Magellanic Clouds (Dordrecht: Kluwer)

Sloan, G. C., Kraemer, K. E., Price, S. D., \& Shipman, R. F. 2003, ApJS, 147, 379

Trams, N. R., van Loon, J. Th., Zijlstra, A. A., et al. 1999a, A\&A, 344, L17

Trams, N. R., van Loon, J. Th., Waters, L. B. F. M., et al. 1999b, A\&A, 346,843

Tsuji, T., Ohnaka, K., Hinkle, K. H., \& Ridgway, S. T. 1994, A\&A, 289,469

Tsuji, T., Ohnaka, K., Aoki, W., \& Yamamura, I. 1997, A\&A, 320, L1

Vandenbussche, B., Beintema, D., \& de Graauw, T., et al. 2002, A\&A, 390,1033

van Loon, J. Th. 2000, A\&A, 354, 125

van Loon, J. Th., Zijlstra, A. A., Bujarrabal, V., \& Nyman, L.-A. 1996, A\&A, 306, L29

van Loon, J. Th., Te Lintel Hekkert, P., Bujarrabal, V., Zijlstra, A. A., \& Nyman, L.-A. 1998a, A\&A, 337, 141

van Loon, J. Th., Zijlstra, A. A., Whitelock, P. A., et al. 1998b, A\&A, 329,169

van Loon, J. Th., Zijlstra, A. A., \& Groenewegen, M. A. T. 1999a, A\&A, 346, 805

van Loon, J. Th., Groenewegen, M. A. T., de Koter, A., et al. 1999b, A\&A, 351, 559

van Loon, J. Th., Zijlstra, A. A., Bujarrabal, V., \& Nyman, L.-A. 2001, A\&A, 368, 950

van Loon, J. Th., Marshall, J. R., Matsuura, M., \& Zijlstra, A. A. 2003, MNRAS, 341, 1205

Vassiliadis, E., \& Wood, P. R. 1993, A\&A, 413, 641

Vermeij, R., Peeters, E., Tielens, A. G. G. M., \& van der Hulst, J. M. 2002, A\&A, 382, 1042

Wallace, L., \& Hinkle, K. 2002, AJ, 124, 3393

Westerlund, B. E., Olander, N., \& Hedin, B. 1981, A\&AS, 43, 267

Whitelock, P. A., Feast, M. W., Menzies, J. W., \& Catchpole, R. M 1989, MNRAS, 238, 769

Whitelock, P. A., Irwin, M., \& Catchpole, R. M. 1996, New Astron., 1,57

Whitelock, P. A., Menzis, J., Irwin, M., \& Feast, M. 1999, IAU Symp., 192, 136

Whitelock, P. A., Feast, M. W., van Loon, J. Th., \& Zijlstra, A. A. 2003, MNRAS, 342, 86

Wood, P. R., Whiteoak, J. B., Hughes, S. M. G., et al. 1992, ApJ, 397, 552

Woods, P. M., Millar, T. J., Zijlstra, A. A., \& Herbst, E. 2002, ApJ, 574, L167

Yamamura, I., de Jong, T., \& Cami, J. 1999b, A\&A, 348, L55

Zijlstra, A. A. 2004, MNRAS, 348, L23

Zijlstra, A. A., Bedding, T. R., Markwick, A. J., et al. 2004, MNRAS, 352,325 